Supporting Information for

\title{
Woven Polymer Networks via the Topological Transformation of a [2]Catenane
}

\author{
Guangfeng Li, ${ }^{\perp}$ Lei Wang, ${ }^{\perp}$ Liang Wu, ${ }^{\perp}$ Zhewen Guo, Jun Zhao, Yuhang Liu, Ruixue Bai, and \\ Xuzhou Yan* \\ ${ }^{\dagger}$ School of Chemistry and Chemical Engineering, Frontiers Science Center for Transformative \\ Molecules, Shanghai Jiao Tong University, Shanghai 200240, P. R. China \\ ${ }^{\perp}$ These authors contributed equally to this work. \\ Email addresses: xzyan@sjtu.edu.cn
}




\section{Materials and methods}

All reagents were commercially available and used as supplied without further purification. All the solvents were used as received from Adamas ${ }^{\circledR}$ beta with a Reagent Grade (99\%). Deuterated solvents were purchased from Cambridge Isotope Laboratory (Andover, MA). All reactions were performed at ambient laboratory conditions, and no precautions are taken to exclude atmospheric moisture unless otherwise specified. Compounds 1-5 were prepared according to the literature procedures. ${ }^{\mathrm{S} 1}$ Notably, the [2]catenane 1 was purified again by recrystallization after column separation and its crystal was used for subsequent characterization and polymerization.

Nuclear magnetic resonance (NMR) spectra were recorded with a Bruker Avance DMX 400 and 500 spectrometers with the use of the deuterated solvent as the lock and the residual solvent or TMS as the internal reference. Solid-state NMR spectra were recorded at ambient pressure on a Bruker AVANCE NEO 600 spectrometer using a standard Bruker magic angle-spinning (MAS) probe with $3.2 \mathrm{~mm}$ (o.d.) zirconia rotors. The magic angle was adjusted by maximizing the number and amplitudes of the signals of the rotational echoes observed in the ${ }^{79} \mathrm{Br}$ MAS FID signal from $\mathrm{KBr}$. The transmitter frequency of ${ }^{13} \mathrm{C}$ NMR is 150 MHz. The solid-state ${ }^{13} \mathrm{C}$ NMR spectra were acquired using cross-polarization (CP) MAS technique with the ninety-degree pulse of ${ }^{1} \mathrm{H}$ with $4.2 \mu$ s pulse width. The $\mathrm{CP}$ contact time was $2 \mathrm{~ms}$.

Inductively coupled plasma optical emission spectroscopy (ICP-OES) was used to determine the zinc contents in these woven materials. Samples were dissolved in OPTIMA grade nitric acid, which were then analyzed on a Thermo-iCAP 7600 Series instrument.

The thermal stability analysis was conducted using a TA Instruments Q500 Thermogravimetric analyzer (TGA) under the nitrogen. Each sample $(\sim 5 \mathrm{mg})$ was heated from 50 to $800{ }^{\circ} \mathrm{C}$ with a rate of $20^{\circ} \mathrm{C} \mathrm{min}^{-1}$.

Fourier transform infrared (FT-IR) spectroscopy was performed on a Thermoscientific Nicolet 6700 FT-IR spectrometer at room temperature in the range of $550 \sim 4000 \mathrm{~cm}^{-1}$.

The solid and solution state fluorescence experiments were conducted using the 
Edinburgh Instruments FLS1000 spectrofluorophotometer. The UV-vis experiments were conducted on a PerkinElmer Lambda 950 absorption spectrophotometer at room temperature.

X-ray diffraction patterns of the [2]catenane and WPN were obtained using a D8 ADVANCE Da Vinci X-Ray Diffractometer (XRD) with $\mathrm{Cu}$ radiation (scan of $2 \theta=5 \sim 30^{\circ}$ with a step size of $0.02^{\circ}$ and count time of $5 \mathrm{sec} / \mathrm{step}$ ). The crystals data of the [2]catenane were collected on an Oxford Diffraction Xcalibur Atlas Gemini ultra.

Dynamic mechanical analysis (DMA) tests under oscillatory stress were performed on rectangular films of the WPN using a TA Instruments DMA Q850 in a tensile mode. The specimens of the WPN were prepared by compression molding to afford self-supporting films that were then cut into rectangular shape. Stress sweep measurement was carried out at room temperature with an oscillation frequency of $1.0 \mathrm{~Hz}$ and an automatic tension setting of $125 \%$. Temperature ramp measurement $\left(-20 \sim 120{ }^{\circ} \mathrm{C}\right)$ at a heating rate of $5^{\circ} \mathrm{C} \min ^{-1}$ was carried out with an oscillation frequency of $1.0 \mathrm{~Hz}$, an oscillation amplitude of $2.0 \mu \mathrm{m}$, and an automatic tension setting of $125 \%$. Frequency sweep measurements $(0.01 \sim 100 \mathrm{~Hz})$ in a logarithmic sweep mode were carried out at room temperature with an oscillation strain of $0.01 \%$, and an automatic tension setting of $125 \%$. Stress-strain experiments were performed in a constant strain mode with a deformation rate of $5.0 \mathrm{~mm} \mathrm{~min}^{-1}$ at room temperature.

Atomic force microscope (AFM) nanomechanical mapping measurements were performed in a PeakForce QNM (Quantitative Nano-Mechanics) mode on a Bruker Bio-FastScan AFM at ambient conditions. The samples of the WPN and demetaleted WPN were prepared through Leica EM UC7 Cryo-ultramicrotome. The moduli and adhesion forces were measured by approaching the tip to the samples and then retracting the tip. The oscillation frequency of the Z-piezo was $2.0 \mathrm{kHz}$, scan rate was $1.0 \mathrm{~Hz}$ and the peak force amplitude was set at $50 \mathrm{~nm}$. The samples were scanned using OMCL-AC240TS-R3 cantilevers (Olympus Micro Cantilevers) with a nominal spring constant of $2.0 \mathrm{~N} \mathrm{~m}^{-1}$. 


\section{Synthesis of [2]catenane 1}

2.1 Synthesis route of [2] catenane 1
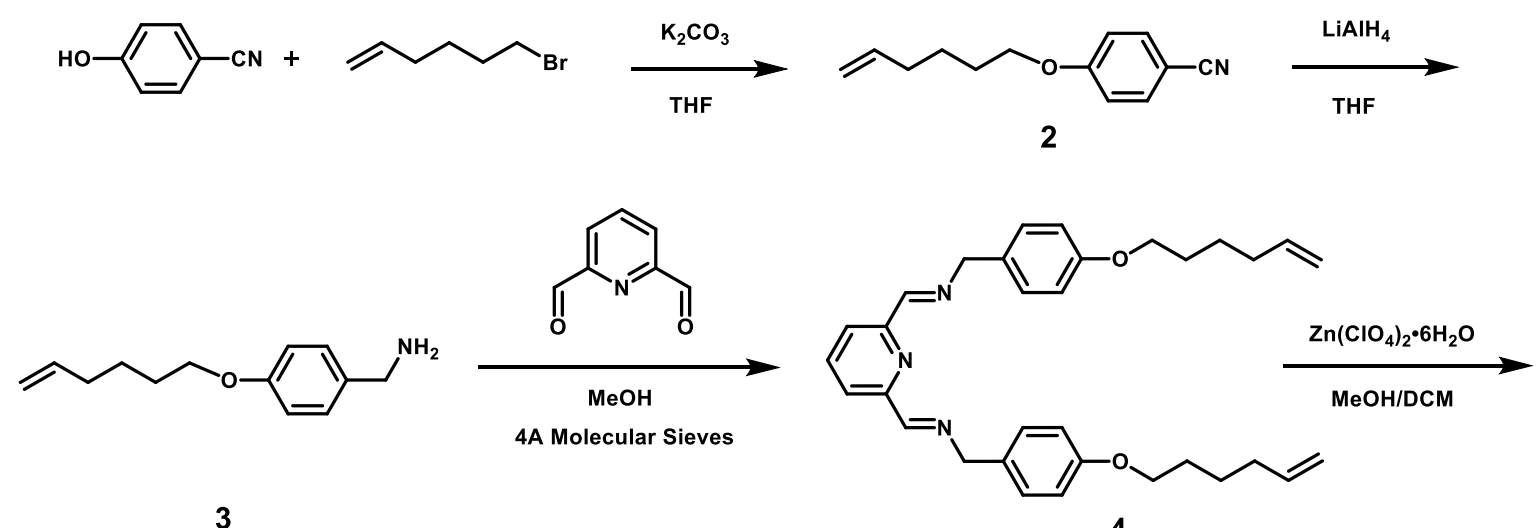

3

4

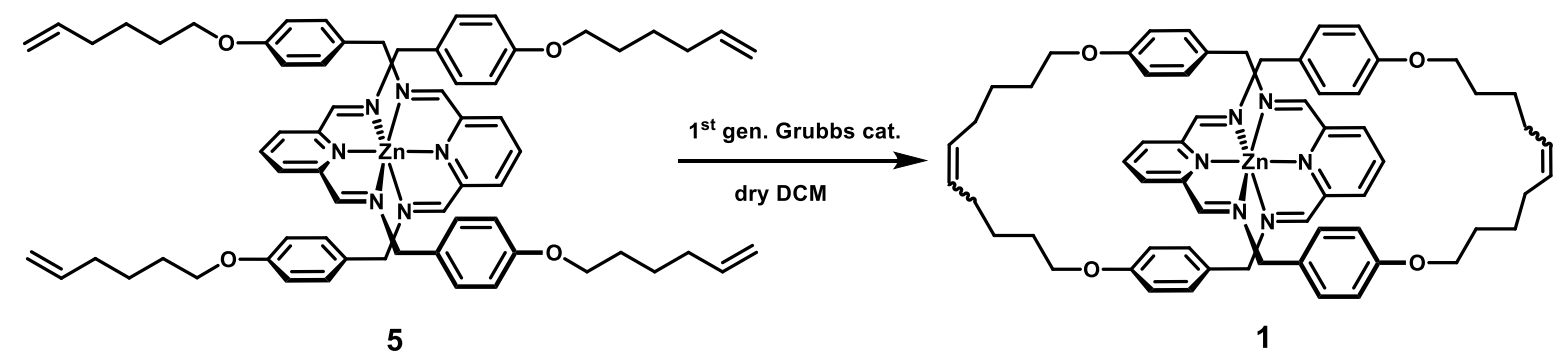

Scheme S1. Synthetic route of the [2]catenane $1 .^{\text {S1 }}$

\subsection{Synthesis of compound 2}

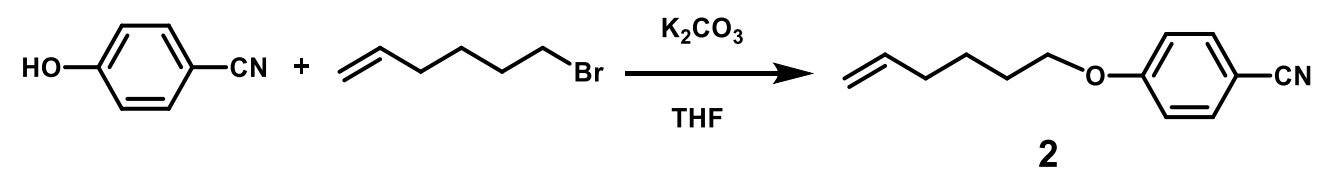

Into a $500 \mathrm{~mL}$ round-bottomed flask were added 4-hydroxybenzonitrile (10.0 g, $84.0 \mathrm{mmol})$, 6-bromo-1-hexene (13.4 g, $84.0 \mathrm{mmol})$, potassium carbonate (11.6 g, $84.0 \mathrm{mmol})$ and $250 \mathrm{~mL}$ of tetrahydrofuran. After heating at reflux for $48 \mathrm{~h}$, water was added to quench the reaction. After removal of tetrahydrofuran, the organic phases were combined, washed with water, brine, and then dried by $\mathrm{Na}_{2} \mathrm{SO}_{4}$ overnight. After filtration and solvent evaporation, 
compound 2 was obtained as a pale yellow oil (15.9 g, 94\%). The ${ }^{1} \mathrm{H}$ NMR spectrum of 2 is shown in Figure $\mathrm{S} 1 .{ }^{1} \mathrm{H} \mathrm{NMR}\left(400 \mathrm{MHz}, \mathrm{CDCl}_{3}\right.$, room temperature) $\delta(\mathrm{ppm}): 7.55$ (d, $J=8.0$ $\mathrm{Hz}, 2 \mathrm{H}), 6.9 .2(\mathrm{~d}, J=8.0 \mathrm{~Hz}, 2 \mathrm{H}), 5.84-5.74(\mathrm{~m}, 1 \mathrm{H}), 5.12-4.74(\mathrm{~m}, 2 \mathrm{H}), 3.99$ (t, $J=6.4 \mathrm{~Hz}$, 2H), 2.15-2.09 (m, 2H), 1.84-1.77(m, 2H), 1.60-1.52 (m, 2H).

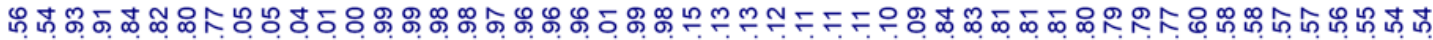

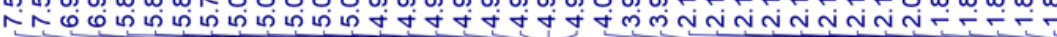
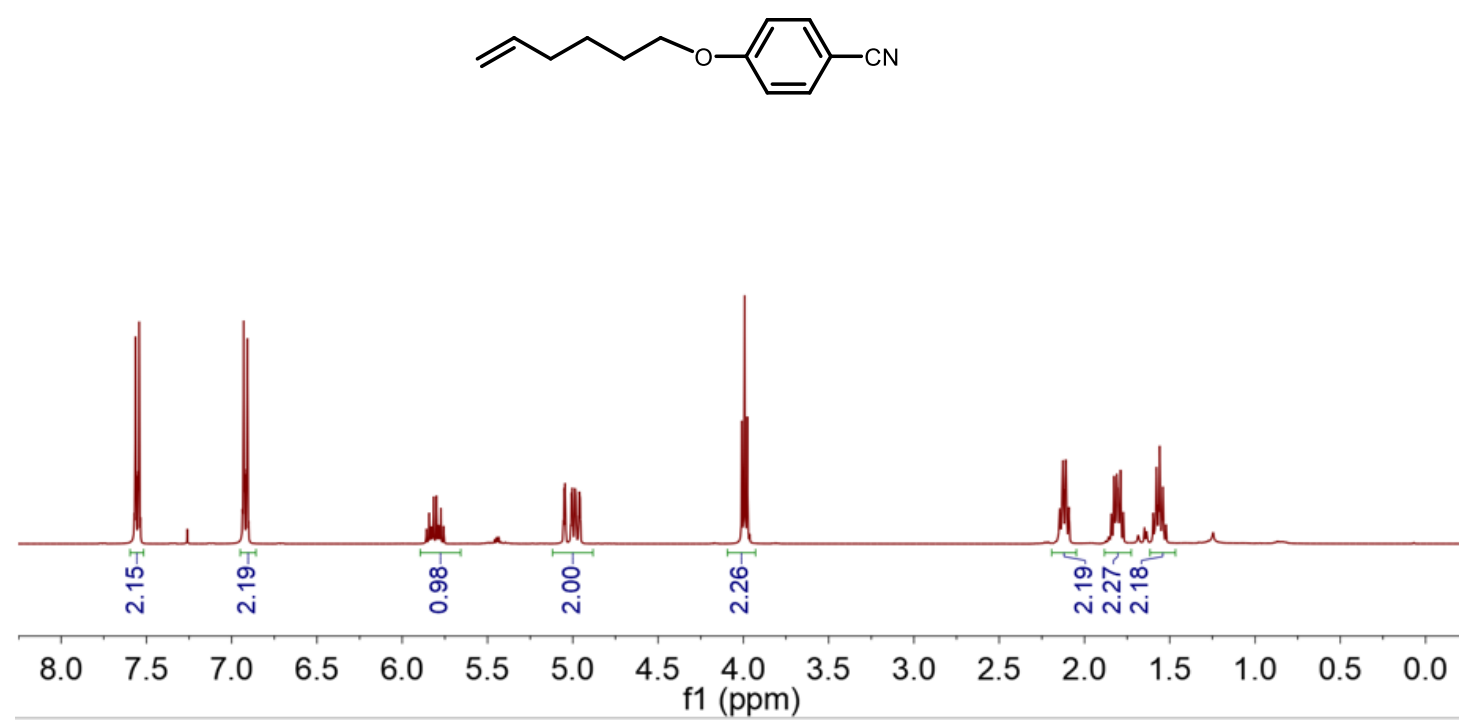

Figure S1. ${ }^{1} \mathrm{H}$ NMR spectrum (400 $\mathrm{MHz}, \mathrm{CDCl}_{3}$, room temperature) of 2.

\section{3 synthesis of compound 3}

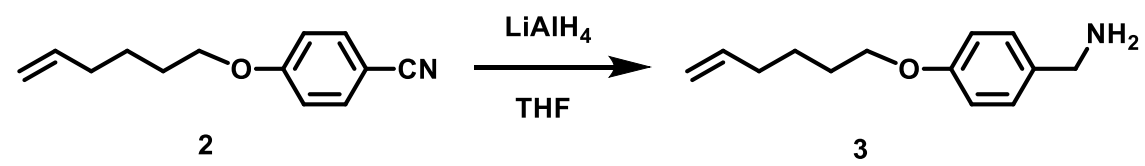

To a solution of $2(10.0 \mathrm{~g}, 50.0 \mathrm{mmol})$ in tetrahydrofuran $(250 \mathrm{~mL})$ was added $\mathrm{LiAlH}_{4}(5.70 \mathrm{~g}$, $150 \mathrm{mmol}$ ) at $0{ }^{\circ} \mathrm{C}$ for 10 minutes. After being stirred at room temperature for $12 \mathrm{~h}$, the reaction mixture was quenched with water and then extracted with dichloromethane. The organic layer was dried by $\mathrm{Na}_{2} \mathrm{SO}_{4}$ overnight. After filtration and solvent evaporation, the 
residue was purified by chromatography over silica gel (eluent: $\mathrm{CH}_{2} \mathrm{Cl}_{2} /$ methanol, 100:1 v/v) to afford $\mathbf{3}(8.52 \mathrm{~g}, 83 \%)$ as a white solid. The ${ }^{1} \mathrm{H}$ NMR spectrum of $\mathbf{3}$ is shown in Figure S2. ${ }^{1} \mathrm{H}$ NMR (400 MHz, $\mathrm{CDCl}_{3}$, room temperature) $\delta(\mathrm{ppm}): 7.20(\mathrm{~d}, J=8.0 \mathrm{~Hz}, 2 \mathrm{H}), 6.85(\mathrm{~d}, J=$ $8.0 \mathrm{~Hz}, 2 \mathrm{H}), 5.90-5.74(\mathrm{~m}, 1 \mathrm{H}), 5.06-4.92(\mathrm{~m}, 2 \mathrm{H}), 3.94(\mathrm{t}, J=6.4 \mathrm{~Hz}, 2 \mathrm{H}), 3.78(\mathrm{~s}, 2 \mathrm{H})$, $2.12(\mathrm{dd}, J=14.3,7.2 \mathrm{~Hz}, 2 \mathrm{H}), 1.87-1.72(\mathrm{~m}, 2 \mathrm{H}), 1.63-1.50(\mathrm{~m}, 2 \mathrm{H})$.

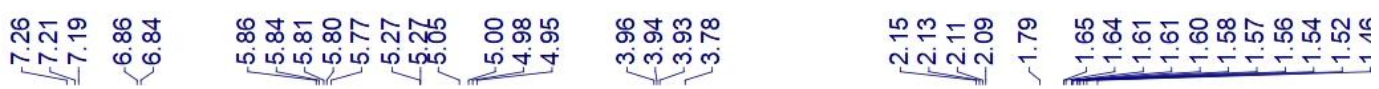
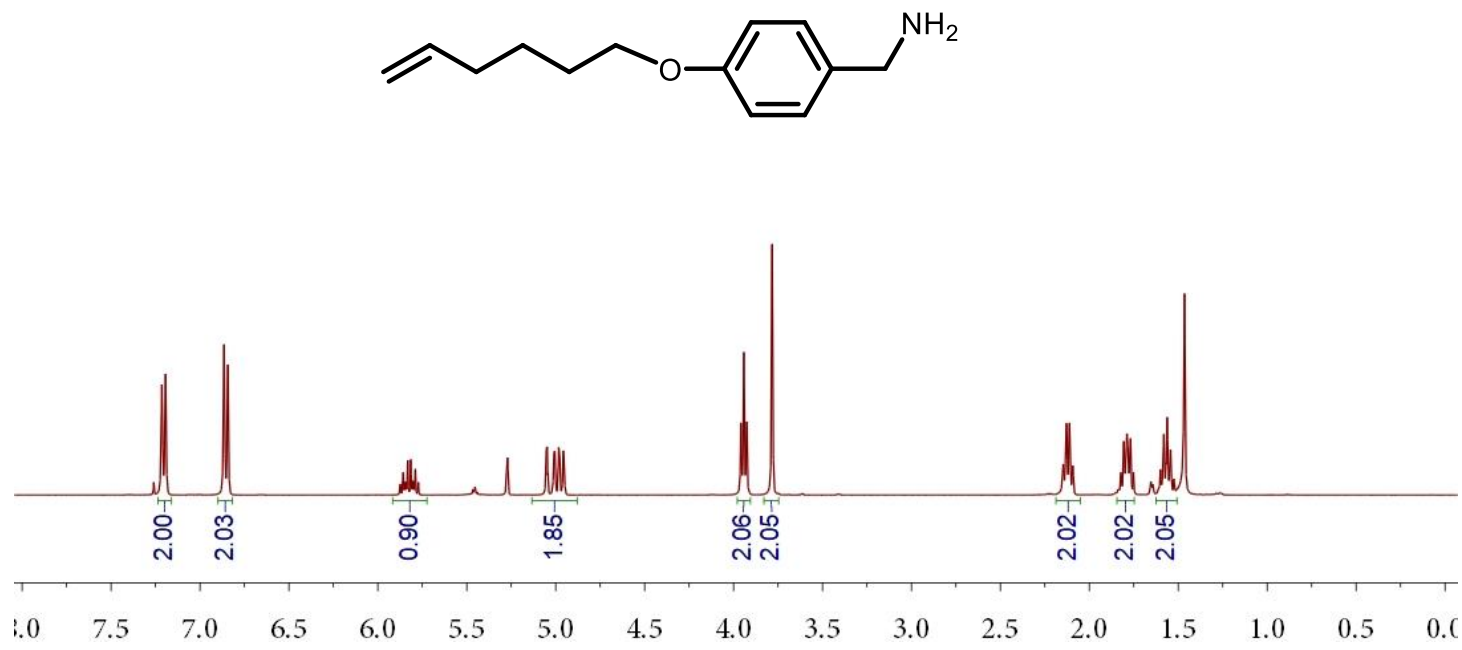

Figure S2. ${ }^{1} \mathrm{H}$ NMR spectrum $\left(400 \mathrm{MHz}, \mathrm{CDCl}_{3}\right.$, room temperature) of 3. 


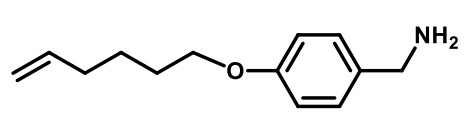

3

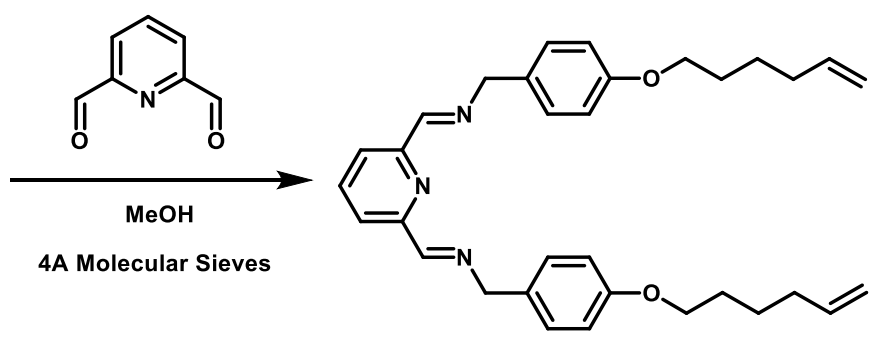

4

To a solution of 2,6-pyridinedicarboxaldehyde (2.10 g, $15.5 \mathrm{mmol})$ in methanol $(200 \mathrm{~mL})$ was added a methanol $(50 \mathrm{~mL})$ solution of $\mathbf{3}(7.00 \mathrm{~g}, 34.1 \mathrm{mmol})$. To the result solution was added $4 \mathrm{~A}$ molecular sieves $(10.0 \mathrm{~g})$. After being stirred at room temperature for $6 \mathrm{~h}$, the reaction mixture was filtered. The solid was collected and washed with $\mathrm{CH}_{2} \mathrm{Cl}_{2}$ for three times, compound 4 (5.71 g, 72\%) was obtained as a white solid. The ${ }^{1} \mathrm{H}$ NMR spectrum of 4 is shown in Figure S3. ${ }^{1} \mathrm{H}$ NMR (400 $\mathrm{MHz}, \mathrm{CDCl}_{3}$, room temperature) $\delta(\mathrm{ppm}): 8.48(\mathrm{~s}, 2 \mathrm{H})$, $8.06(\mathrm{~d}, J=7.6 \mathrm{~Hz}, 2 \mathrm{H}), 7.78(\mathrm{t}, J=7.6 \mathrm{~Hz}, 1 \mathrm{H}), 7.28-7.20(\mathrm{~m}, 4 \mathrm{H}), 6.88(\mathrm{~d}, J=8.4 \mathrm{~Hz}, 4 \mathrm{H})$, 5.93-5.73 (m, 2H), 5.13-4.91 (m, 4H), $4.84(\mathrm{t}, J=6.8 \mathrm{~Hz}, 4 \mathrm{H}), 4.05-3.90(\mathrm{~m}, 4 \mathrm{H}), 2.18-2.07$ (m, 4H), 1.89-1.74 (m, 4H), 1.53-1.50 (m, 4H). 

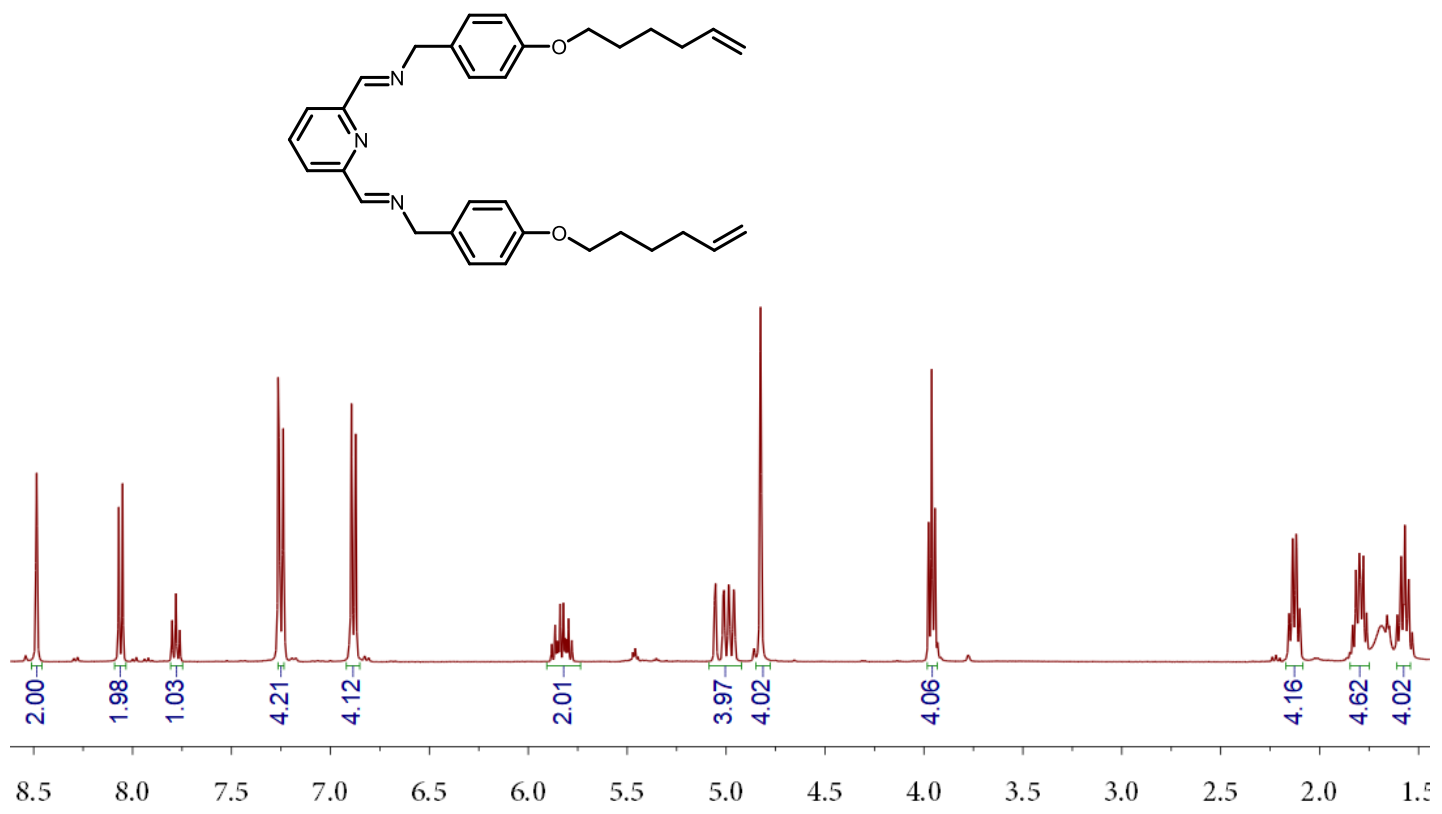

Figure S3. ${ }^{1} \mathrm{H}$ NMR spectrum (400 $\mathrm{MHz}, \mathrm{CDCl}_{3}$, room temperature) of 4.

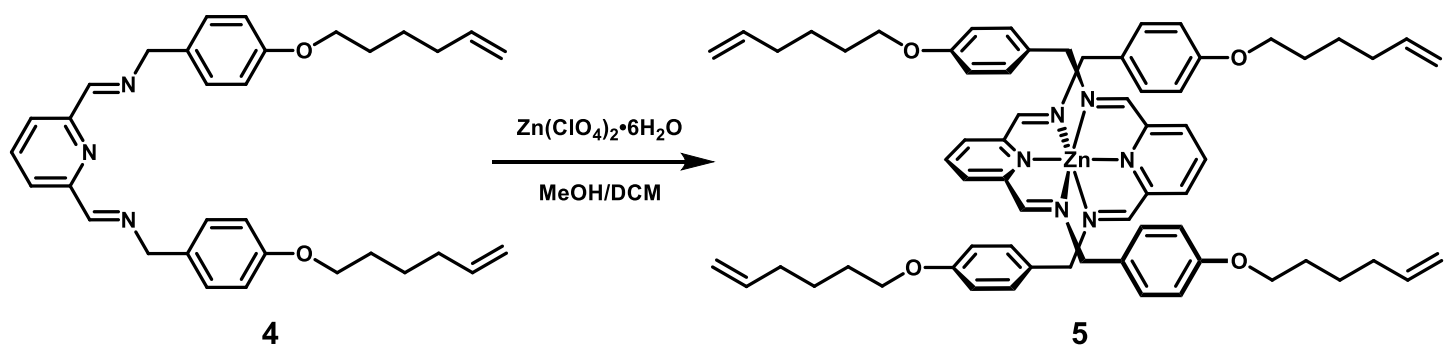

To a solution of $\mathrm{ZnClO}_{4} \cdot 6 \mathrm{H}_{2} \mathrm{O}(1.82 \mathrm{~g}, 4.90 \mathrm{mmol})$ in methanol $(100 \mathrm{~mL})$ was added a solution of compound $4(5.00 \mathrm{~g}, 9.80 \mathrm{mmol})$ in $\mathrm{CH}_{2} \mathrm{Cl}_{2}(200 \mathrm{~mL})$. After being stirred at room temperature for $12 \mathrm{~h}$, the reaction mixture was concentrated. The residue was collected and washed with methanol for three times, compound $5(4.30 \mathrm{~g}, 81 \%)$ was obtained as a white solid. The ${ }^{1} \mathrm{H}$ NMR spectrum of 5 is shown in Figure S4. ${ }^{1} \mathrm{H}$ NMR (400 MHz, DMSO- $d_{6}$, room temperature) $\delta(\mathrm{ppm}): 8.58-8.50(\mathrm{~m}, 2 \mathrm{H}), 8.32(\mathrm{~s}, 4 \mathrm{H}), 8.02(\mathrm{~d}, J=7.6 \mathrm{~Hz}, 4 \mathrm{H}), 6.50(\mathrm{~s}$, $16 \mathrm{H}), 5.91-5.79(\mathrm{~m}, 4 \mathrm{H}), 5.09-4.92(\mathrm{~m}, 8 \mathrm{H}), 4.26(\mathrm{~s}, 8 \mathrm{H}), 3.90(\mathrm{t}, J=6.4 \mathrm{~Hz}, 8 \mathrm{H}), 2.16-2.06$ (m, 8H), 1.80-1.66 (m, 8H), 1.60-1.45 (m, 8H). 


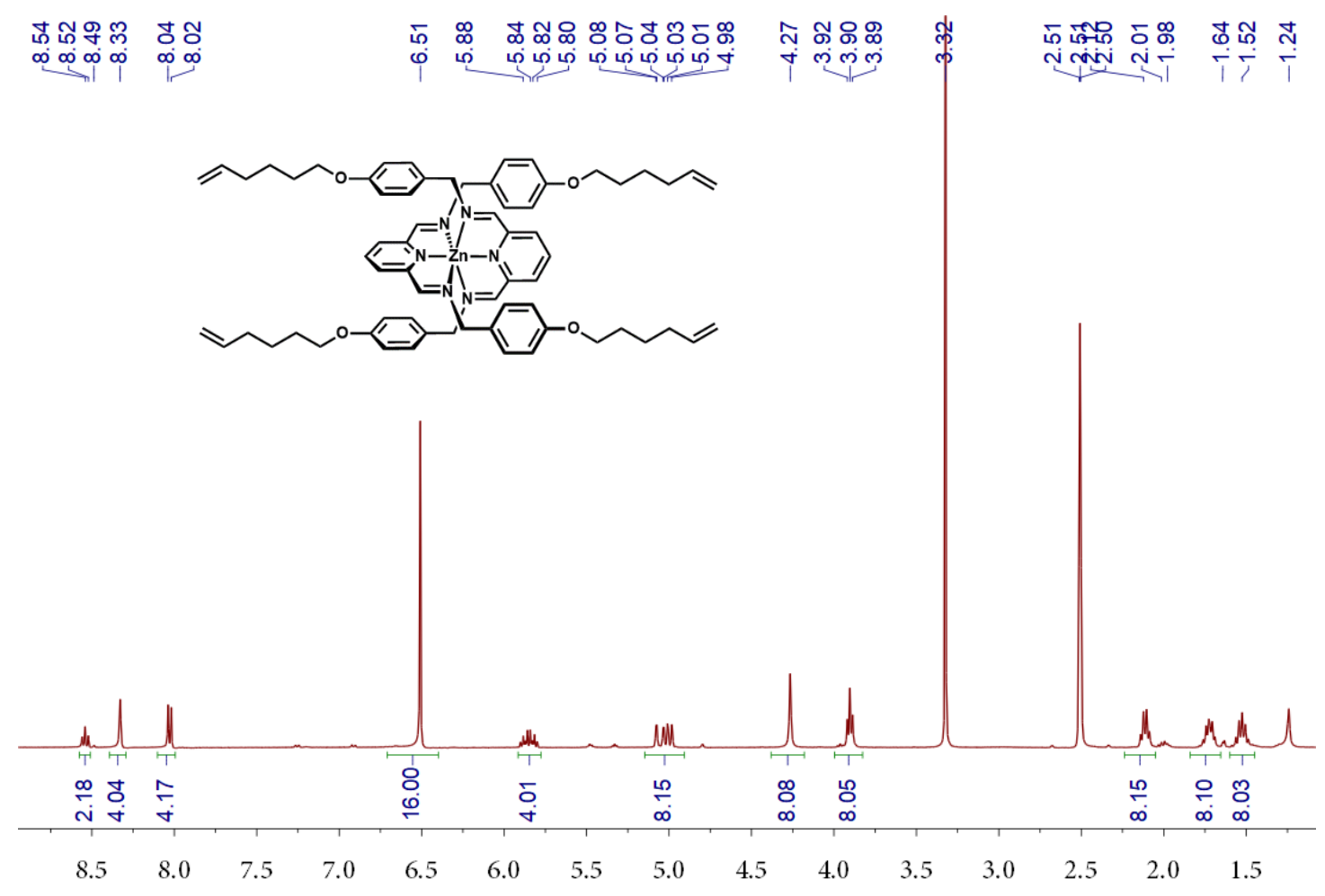

Figure S4. ${ }^{1} \mathrm{H}$ NMR spectrum (400 MHz, DMSO-d6, room temperature) of 5.

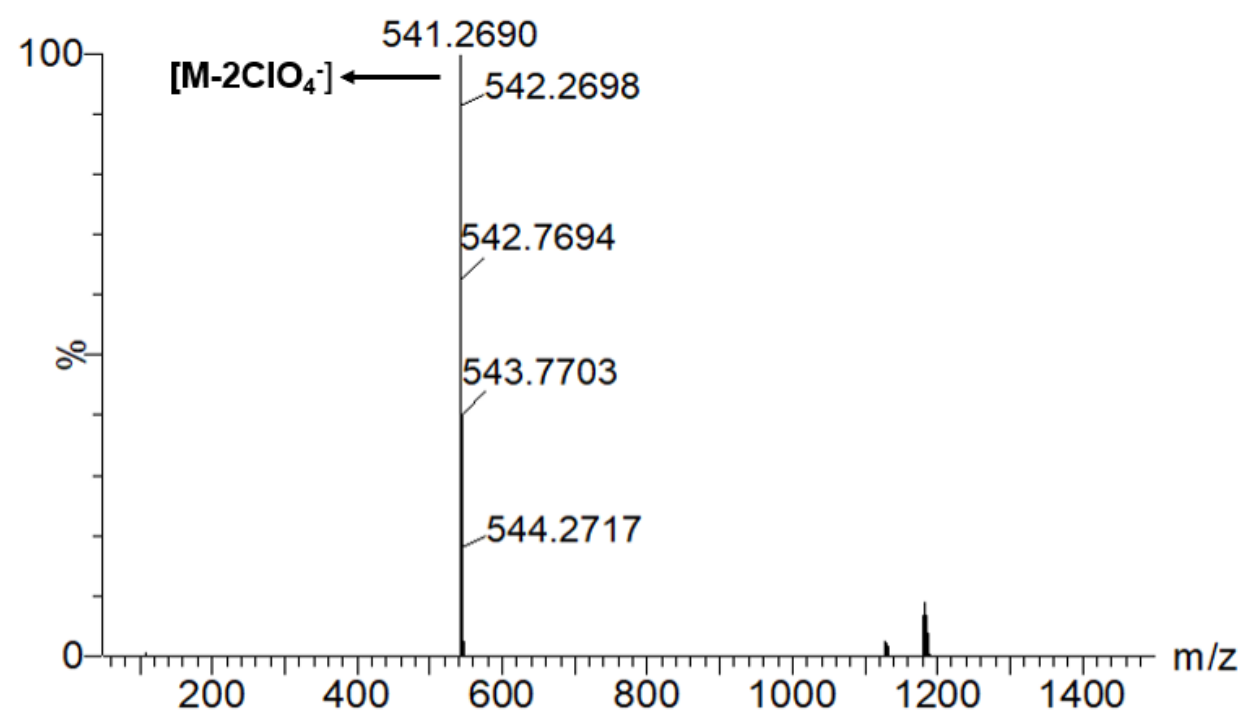

Figure S5. Electrospray ionization mass spectrum of $\mathbf{5}$. 


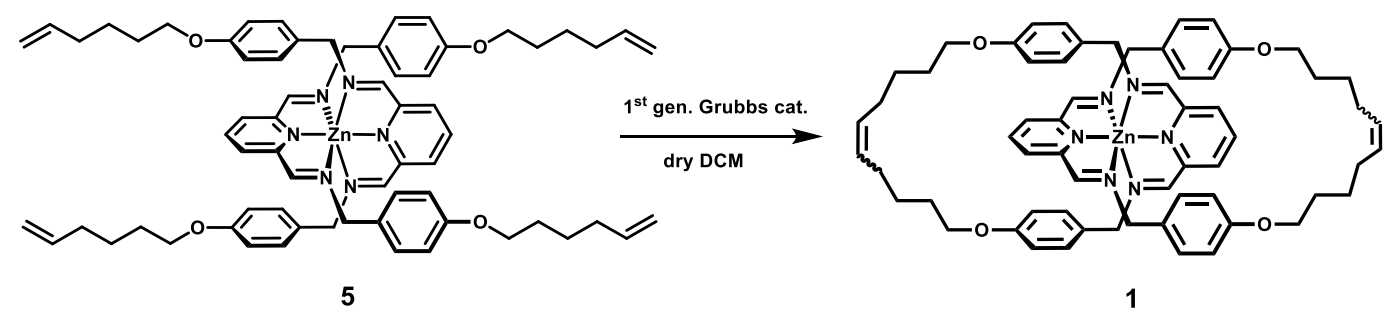

A solution of $5(250 \mathrm{mg}, 0.10 \mathrm{mmol})$ in anhydrous $\mathrm{CH}_{2} \mathrm{Cl}_{2}(300 \mathrm{~mL})$ was purged with $\mathrm{N}_{2}$ for $10 \mathrm{~min}$. Then, a solution of Grubbs I catalyst (20 mg, $10 \mathrm{~mol} \%)$ in $\mathrm{CH}_{2} \mathrm{Cl}_{2}(10 \mathrm{~mL})$ was added by syringe and the reaction mixture was heated at $30{ }^{\circ} \mathrm{C}$ for 4 days under $\mathrm{N}_{2}$. The solvent was evaporated off under reduced pressure and the crude product was purified by chromatography over silica gel (eluent: $\mathrm{CH}_{2} \mathrm{Cl}_{2} /$ methanol, 10:1 v/v) to afford $\mathbf{1}(70 \mathrm{mg}, 34 \%)$ as a brown solid. The ${ }^{1} \mathrm{H}$ NMR spectrum of $\mathbf{1}$ is shown in Figure S6. ${ }^{1} \mathrm{H}$ NMR $\left(400 \mathrm{MHz}\right.$, DMSO- $d_{6}$, room temperature) $\delta(\mathrm{ppm}): 8.50(\mathrm{~s}, 4 \mathrm{H}), 8.43-8.39(\mathrm{~m}, 2 \mathrm{H}), 7.70(\mathrm{~d}, J=7.6 \mathrm{~Hz}, 4 \mathrm{H}), 6.57-6.48(\mathrm{~m}$, $8 \mathrm{H}), 6.36(\mathrm{~d}, J=8.4 \mathrm{~Hz}, 8 \mathrm{H}), 5.60(\mathrm{t}, J=3.6 \mathrm{~Hz}, 4 \mathrm{H}), 4.54-4.42(\mathrm{~m}, 8 \mathrm{H}), 3.85(\mathrm{t}, J=6.4 \mathrm{~Hz}$, $8 \mathrm{H}), 2.12(\mathrm{~s}, 8 \mathrm{H}), 1.80-1.67(\mathrm{~m}, 8 \mathrm{H}), 1.61-1.55(\mathrm{~m}, 8 \mathrm{H})$. The ${ }^{13} \mathrm{C}$ NMR spectrum of $\mathbf{1}$ is shown in Figure S7. ${ }^{13} \mathrm{C}$ NMR (125 MHz, DMSO- $d_{6}$, room temperature) $\delta$ (ppm): 160.39 , $158.32,145.33,144.46,130.72,130.07,129.43,127.13,113.99,67.25,60.85,31.60,28.14$, 25.99 .

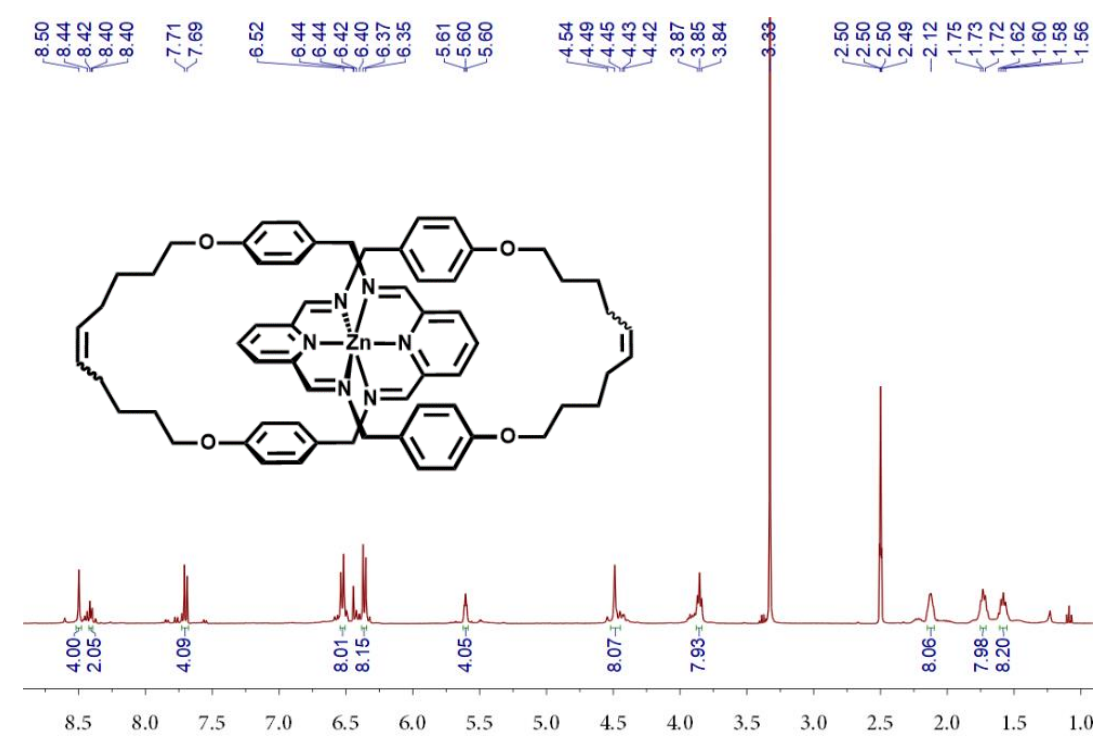


Figure S6. ${ }^{1} \mathrm{H}$ NMR spectrum (400 MHz, DMSO- $d_{6}$, room temperature) of the [2]catenane .

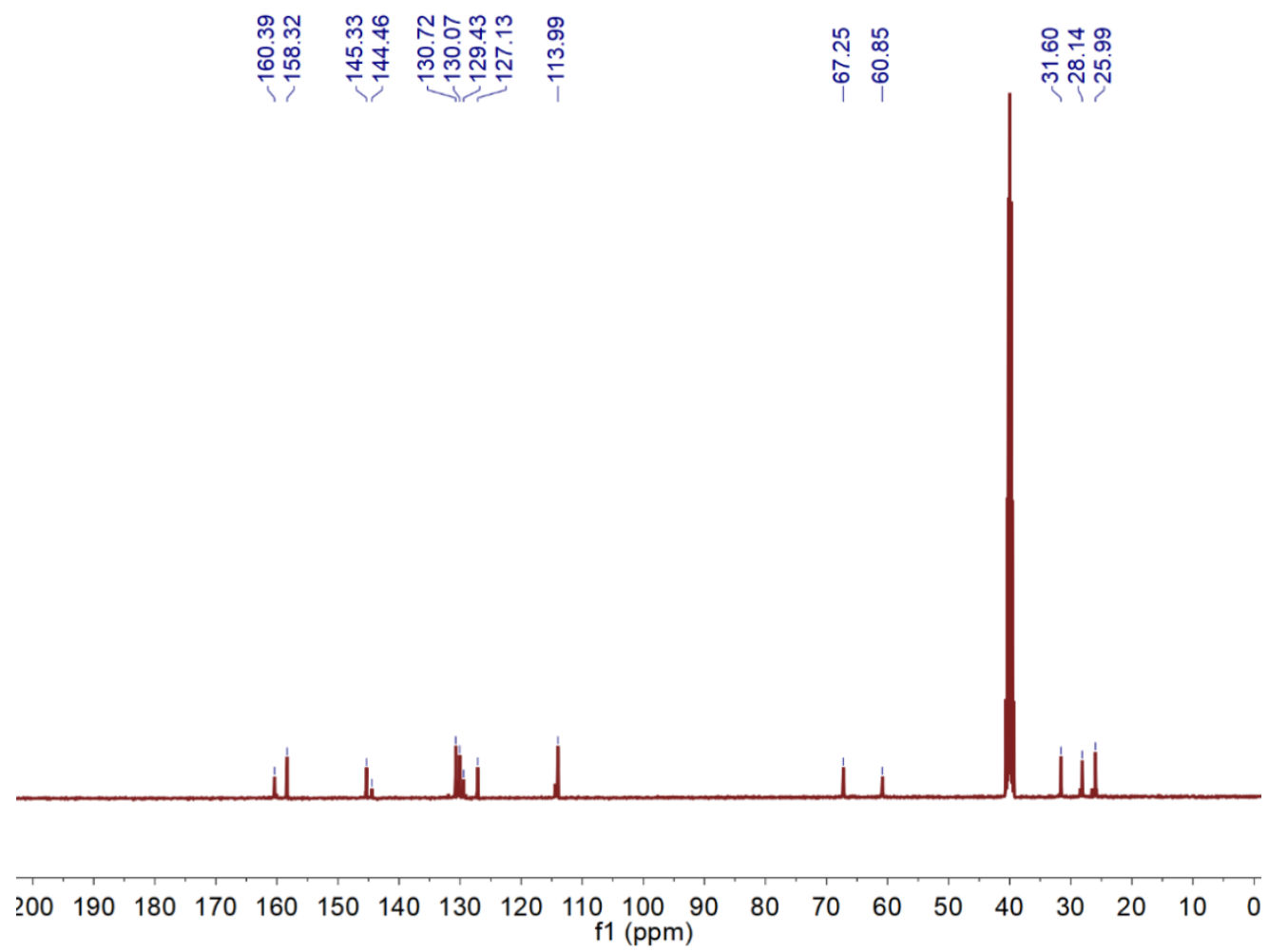

Figure S7. ${ }^{13} \mathrm{C}$ NMR spectrum $\left(125 \mathrm{MHz}, \mathrm{DMSO}-d_{6}\right.$, room temperature) of the [2]catenane.

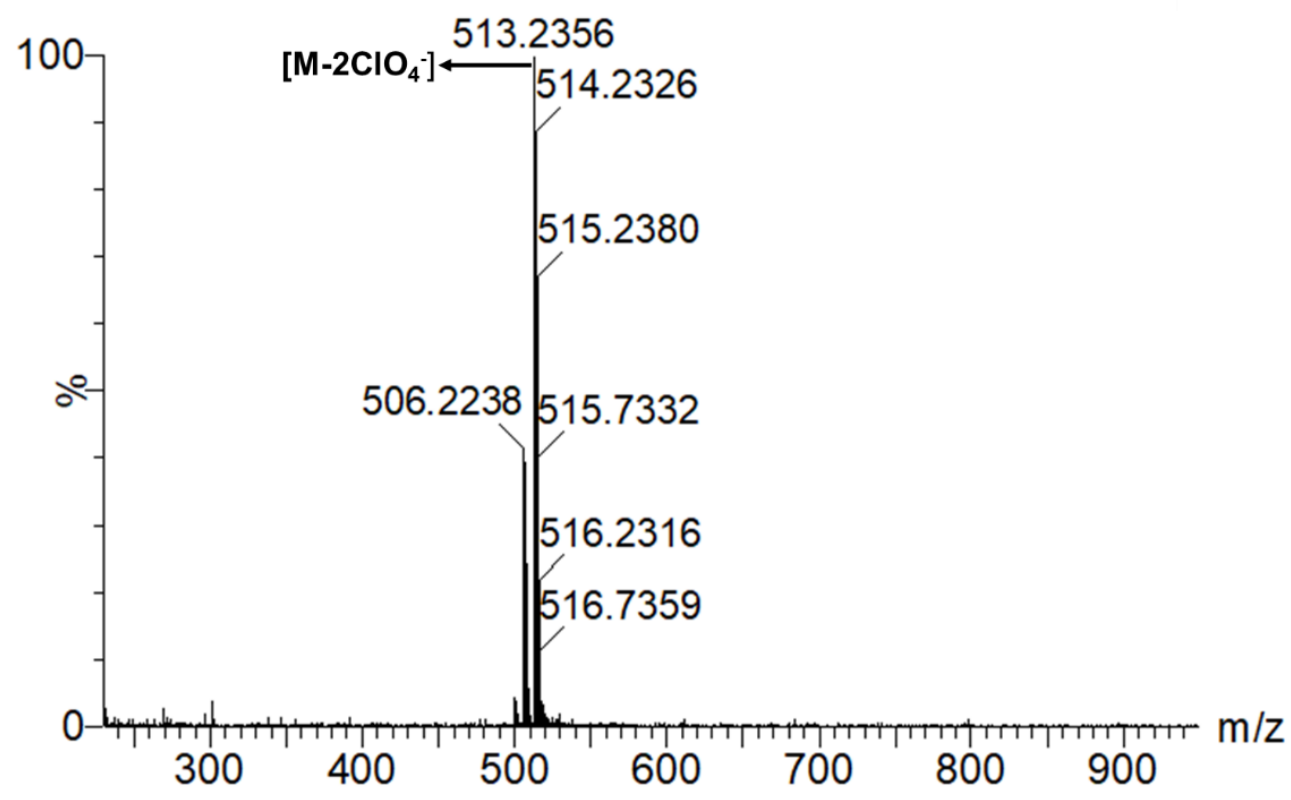


Figure S8. Electrospray ionization mass spectrum of the [2]catenane.

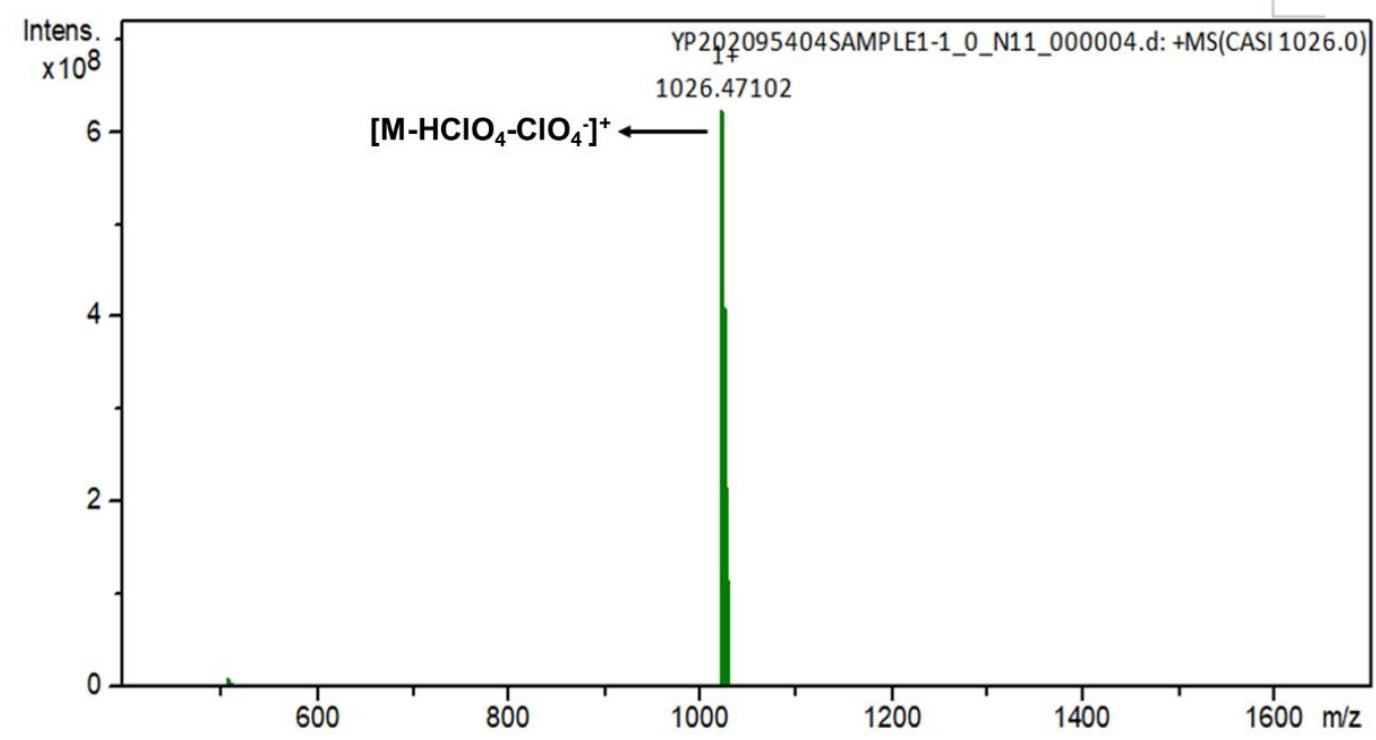

Figure S9. MALDI-TOF MS spectrum of the [2]catenane.

\section{X-Ray Crystallography}

The [2]catenane crystal suitable for X-ray crystallography was obtained by slow vapor diffusion of diethyl ether into a concentrated $\mathrm{CH}_{3} \mathrm{CN}$ solution of the [2]catenane. The [2]catenane crystal structure is in accord with the one reported by Leigh et al (CCDC deposition number: 147870). 


\begin{tabular}{|c|c|}
\hline Compound & [2]catenane \\
\hline Formula & $\mathrm{C}_{62} \mathrm{H}_{70} \mathrm{Cl}_{2} \mathrm{~N}_{6} \mathrm{O}_{12} \mathrm{Zn}$ \\
\hline$D_{\text {calc. }} / \mathrm{g} \mathrm{cm}^{-3}$ & 1.312 \\
\hline$m / \mathrm{mm}^{-1}$ & 1.872 \\
\hline Formula Weight & 1227.51 \\
\hline Colour & colourless \\
\hline Shape & block \\
\hline Size $/ \mathrm{mm}^{3}$ & $0.22 \times 0.20 \times 0.18$ \\
\hline$T / \mathrm{K}$ & $173(2)$ \\
\hline Crystal System & monoclinic \\
\hline Space Group & $P 2_{1} / c$ \\
\hline$a / \AA ̊$ & $10.7010(2)$ \\
\hline$b / \AA$ & $17.4358(3)$ \\
\hline$c / \AA ̊$ & $33.6103(7)$ \\
\hline$a l^{\circ}$ & 90 \\
\hline$b 1^{\circ}$ & $97.8450(10)$ \\
\hline$g l^{\circ}$ & 90 \\
\hline $\mathrm{V} / \AA^{3}$ & $6212.3(2)$ \\
\hline$Z$ & 4 \\
\hline$Z^{\prime}$ & 1 \\
\hline Wavelength/Å & 1.54178 \\
\hline Radiation type & $\mathrm{CuK}_{a}$ \\
\hline$Q_{m i n} I^{\circ}$ & 2.861 \\
\hline$Q_{\max } l^{\circ}$ & 68.391 \\
\hline Measured Refl's. & 51265 \\
\hline Ind't Refl's & 11349 \\
\hline Refl's with I > 2(I) & 7317 \\
\hline$R_{i n t}$ & 0.0465 \\
\hline Parameters & 859 \\
\hline Restraints & 301 \\
\hline Largest Peak & 0.760 \\
\hline Deepest Hole & -0.618 \\
\hline GooF & 1.057 \\
\hline$w R_{2}$ (all data) & 0.2916 \\
\hline$w R_{2}$ & 0.2557 \\
\hline$R_{I}$ (all data) & 0.1116 \\
\hline$R_{1}$ & 0.0832 \\
\hline
\end{tabular}




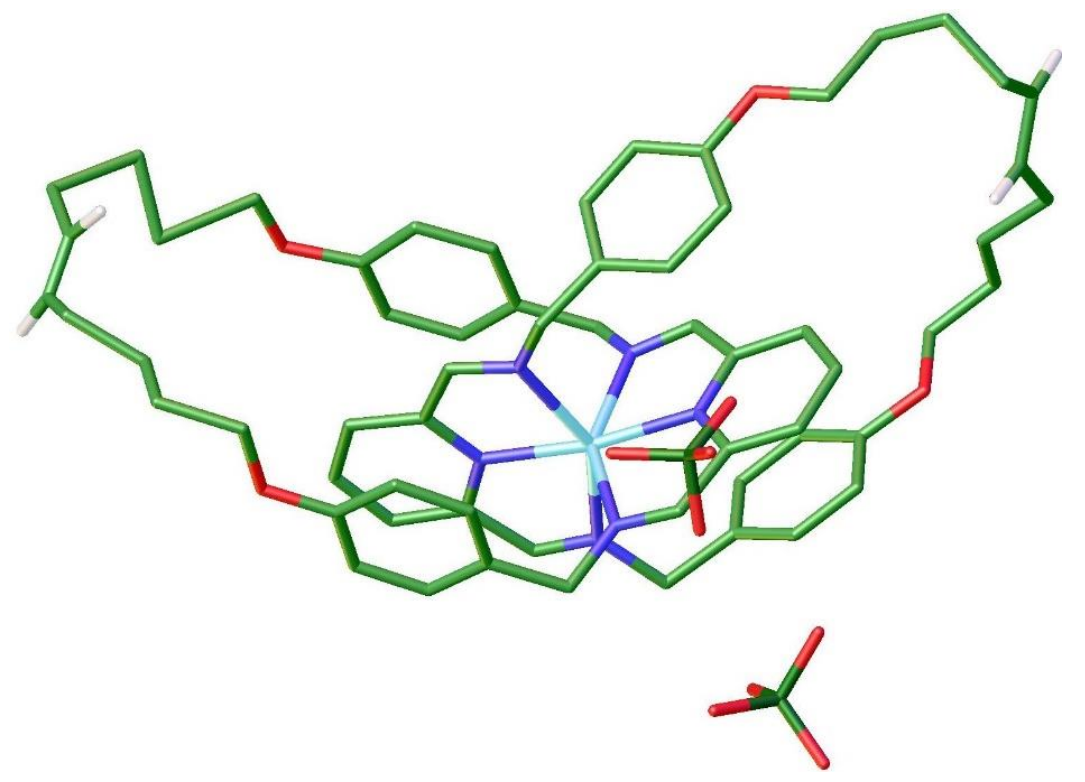

Figure S10. X-ray crystal structure of the [2]catenane.

\section{4. ${ }^{1} \mathrm{H}-{ }^{13} \mathrm{CHSQC}$ spectrum of the [2]catenane}

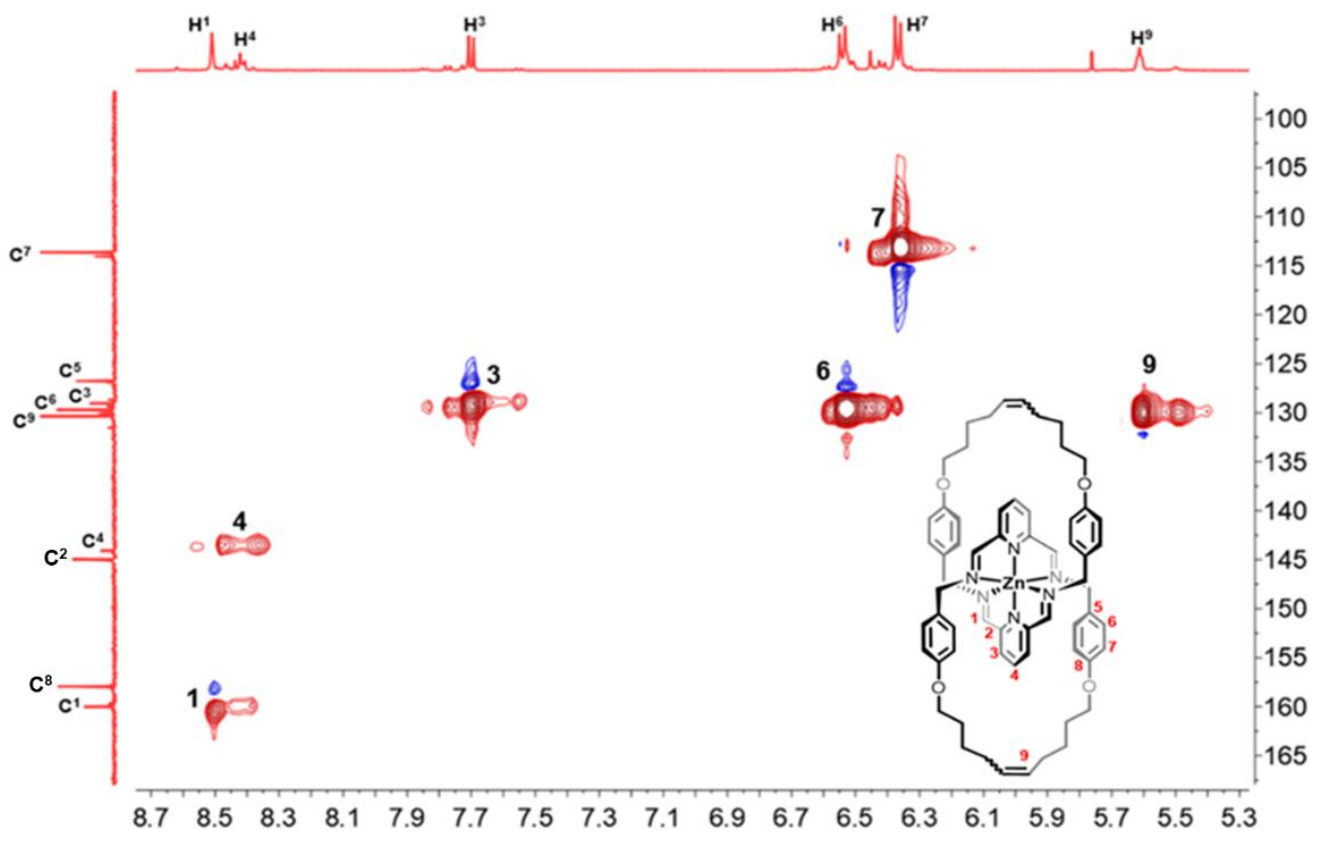

Figure S11. Partial ${ }^{1} \mathrm{H}_{-}{ }^{13} \mathrm{C}$ HSQC spectrum (500 MHz, DMSO- $d_{6}$, room temperature) of the [2]catenane. 


\section{Ring-opening metathesis polymerization of the [2]catenane}
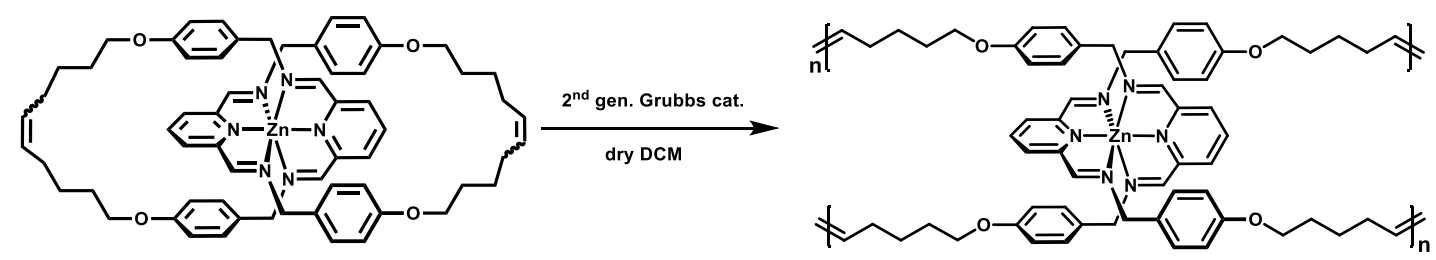

[2]Catenane 1 (100 mg, $0.10 \mathrm{mmol})$ was dissolved in anhydrous DCM (3.0 mL). The solution was fully degassed for $20 \mathrm{~min}$. A solution of Grubbs II catalyst (2.0 mg, $2.0 \mathrm{~mol} \%$ ) in DCM was added to initiate the polymerization at room temperature. After the reaction mixture was stirred at room temperature for $4 \mathrm{~h}$, several drops of ethyl vinyl ether were added to quench the polymerization. The polymer solution was diluted in DCM and precipitated into methanol for six times to yield the WPN. The WPN was collected by filtration, dried under high vacuum at $50{ }^{\circ} \mathrm{C}$ for $3 \mathrm{~h}$, and obtained as an off-white solid (20 mg, 20\%).

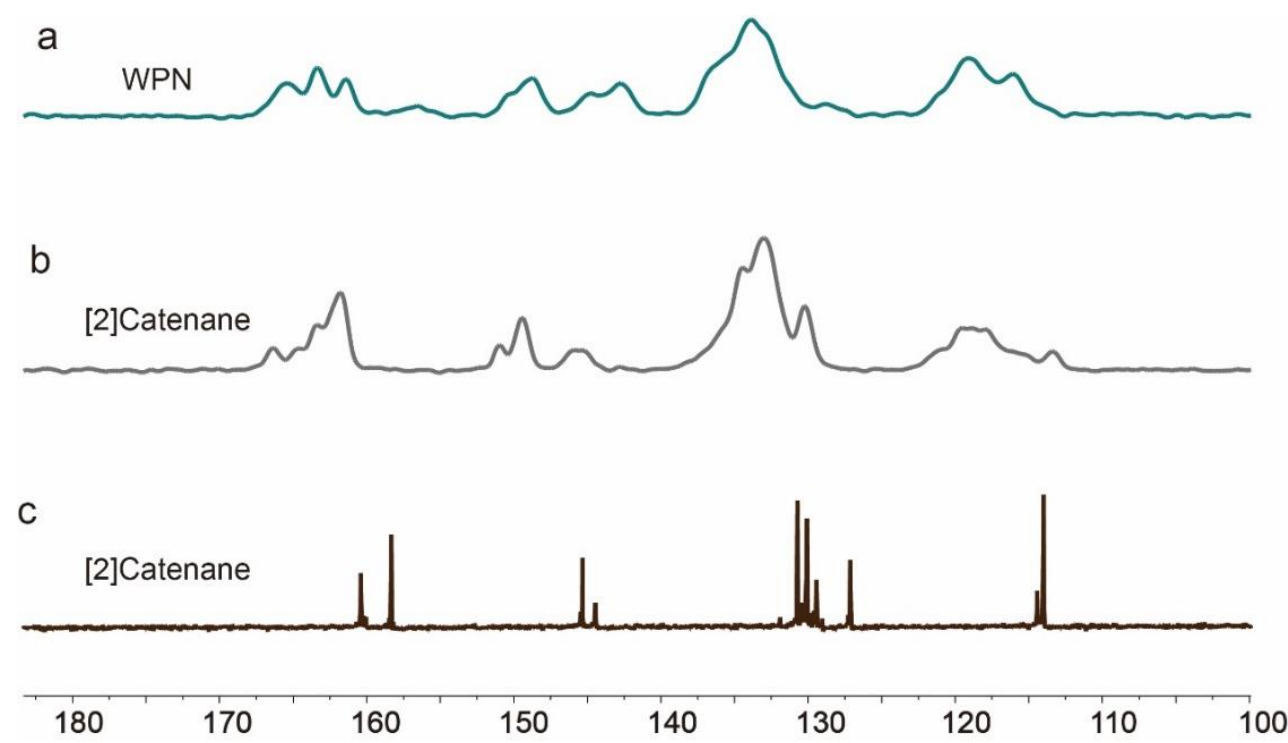

Figure S12. Partial ${ }^{13} \mathrm{C}$ CP/MAS solid state NMR spectra $(150 \mathrm{MHz}$, room temperature ) of the WPN (a), [2]catenane (b) and Partial ${ }^{13} \mathrm{C}$ NMR spectrum (500 $\mathrm{MHz}, \mathrm{DMSO}-d_{6}$, room temperature ) of [2]catenane (c). 


\section{Demetalation and remetalation of the WPN}

6.1 Procedure for demetalation.

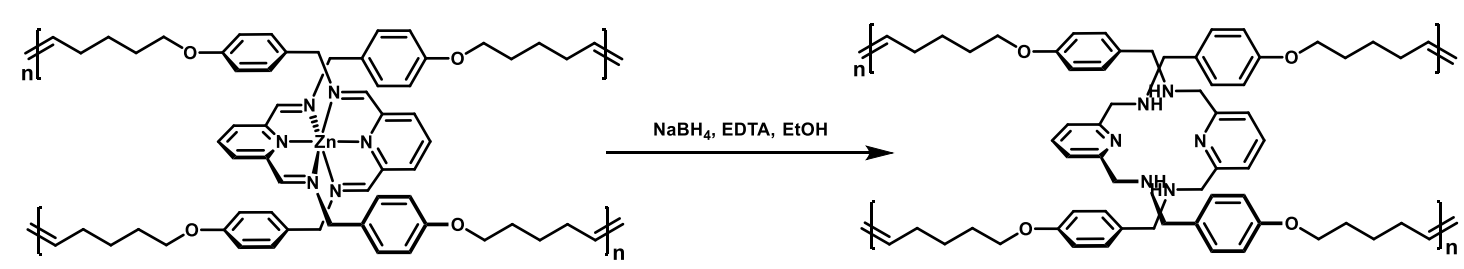

The WPN (20.0 mg) was suspended in ethanol to form a suspension, and then excessive $\mathrm{NaBH}_{4}$ (30.0 eq.) was added portion to the stirring solution over a period of $2 \mathrm{~h}$. Stirring was maintained under ambient conditions for a further $24 \mathrm{~h}$. Then, to this suspension was dropwise added a $0.2 \mathrm{M}$ EDTA aqueous solution $(20.0 \mathrm{~mL})$. The solution was replaced by a fresh solution of EDTA every $2 \mathrm{~h}$ and this procedure was repeated four times. Subsequently, the suspension was filtered and solid was collected then washed with anhydrous $\mathrm{CH}_{3} \mathrm{OH}$ and dried at $120{ }^{\circ} \mathrm{C}$ in oven for $12 \mathrm{~h}$ to produce the demetalated WPN. We referred to the procedure reported by Leigh and coworkers for the demetalation of the WPN. ${ }^{\mathrm{S} 1}$

\subsection{Procedure for remetalation}

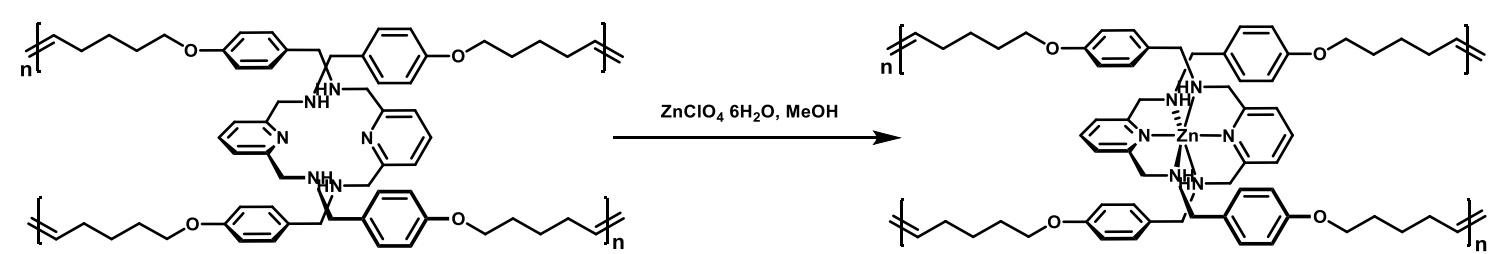

The remetalation process was carried out in similar conditions with the complexation reaction to yield compound 5. Dried demetalated WPN $(20.0 \mathrm{mg})$ was immersed in anhydrous $\mathrm{CH}_{3} \mathrm{OH}$, to which was added a solution of $\mathrm{ZnClO}_{4} \cdot 6 \mathrm{H}_{2} \mathrm{O}(4.0 \mathrm{mg})$ in methanol. This mixture was stirred for $12 \mathrm{~h}$ at room temperature, and the solid was collected and washed with anhydrous $\mathrm{CH}_{3} \mathrm{OH}$. After the solid was dried at $120{ }^{\circ} \mathrm{C}$ in oven for $12 \mathrm{~h}$, the remetalated WPN was 
obtained. We referred to the procedure reported by Leigh and coworkers for the remetalation of the demetalated WPN. ${ }^{\mathrm{S} 1}$

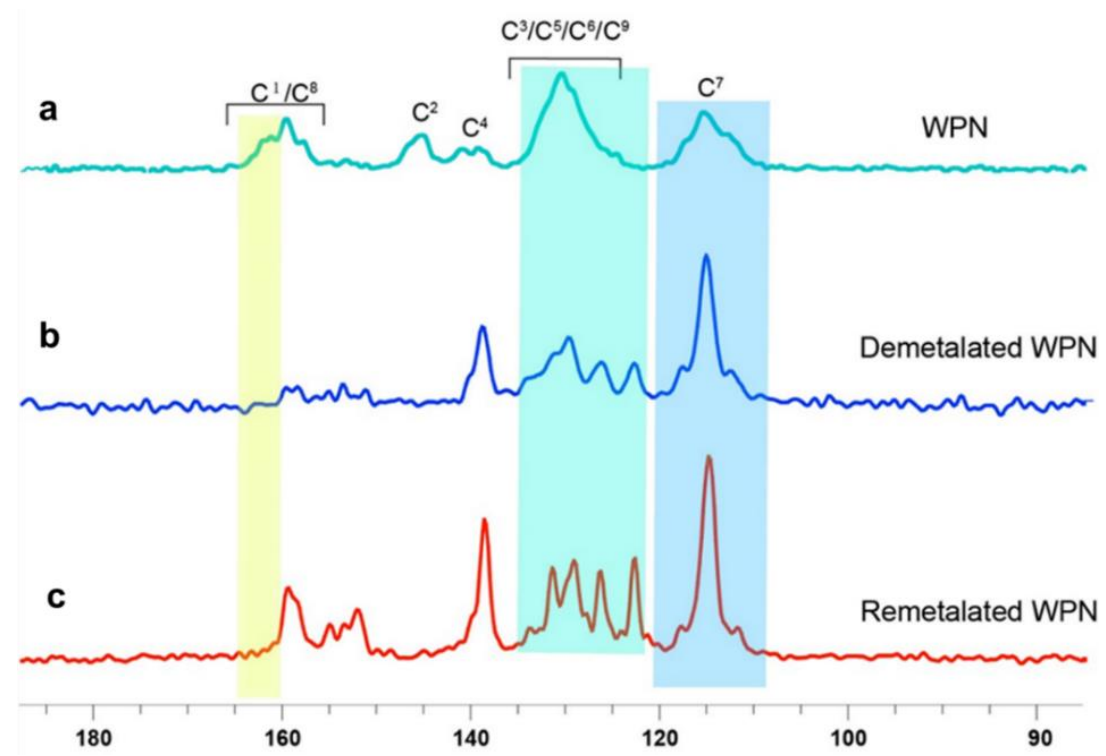

Figure S13. Partial ${ }^{13} \mathrm{C}$ CP/MAS solid state NMR spectra $(150 \mathrm{MHz}$, room temperature) of the WPN (a), demetalated WPN (b) and remetalated WPN (c). 


\section{a}

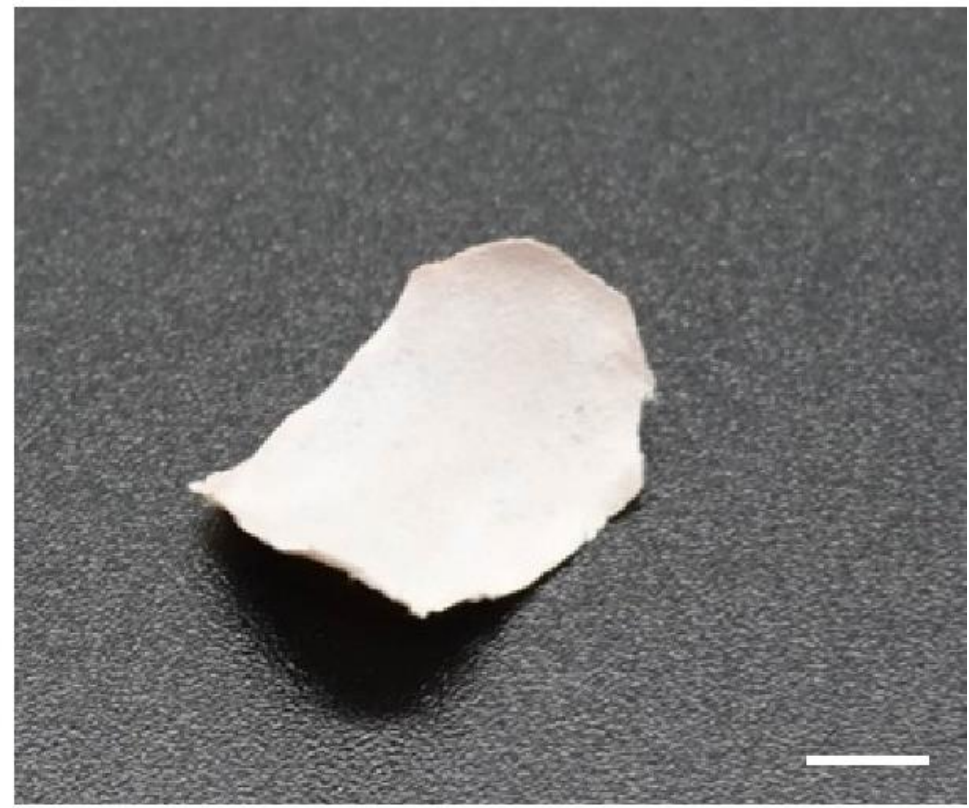

b

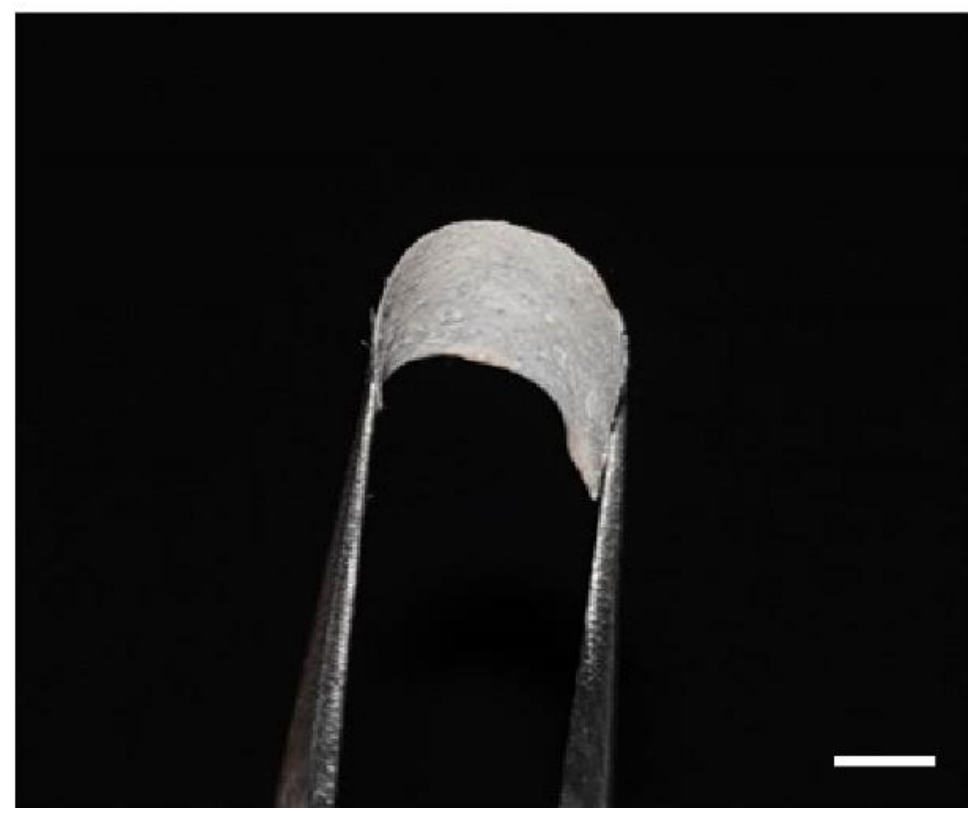

Figure S14. Photographs of the WPN (scale bar $=5 \mathrm{~mm}$ ). 


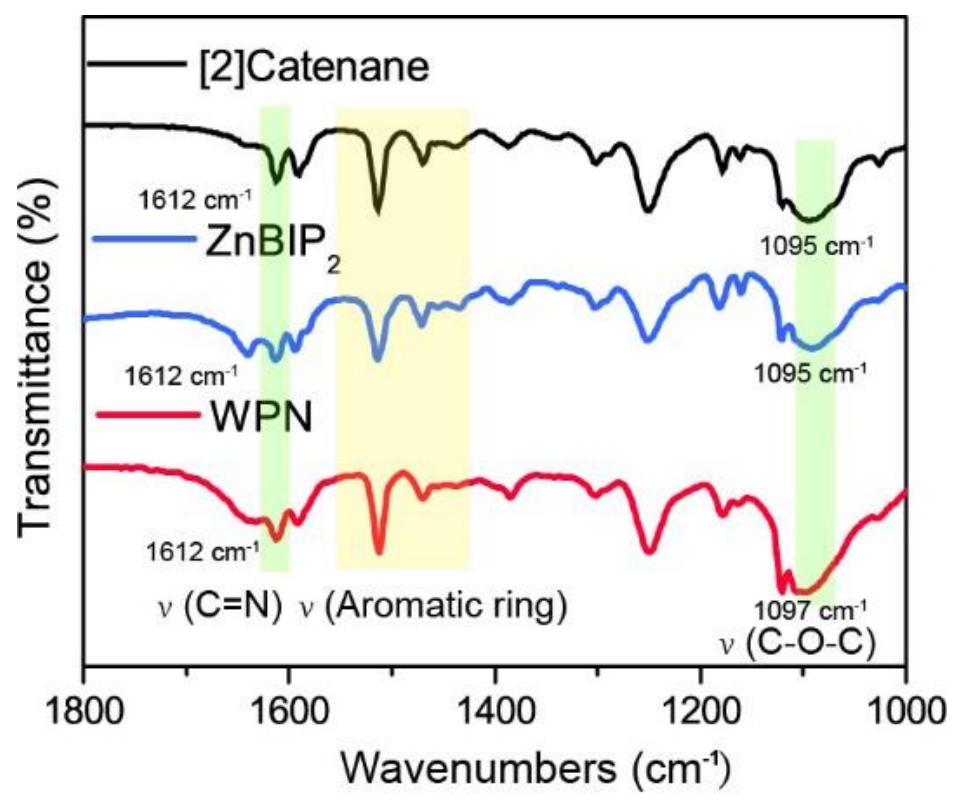

Figure S15. Partial FT-IR spectra of the [2]catenane, $\mathrm{ZnBIP}_{2}$ and WPN.

9. TGA curves of the [2]catenane, $\mathrm{ZnBIP}_{2}$ and $\mathrm{WPN}$

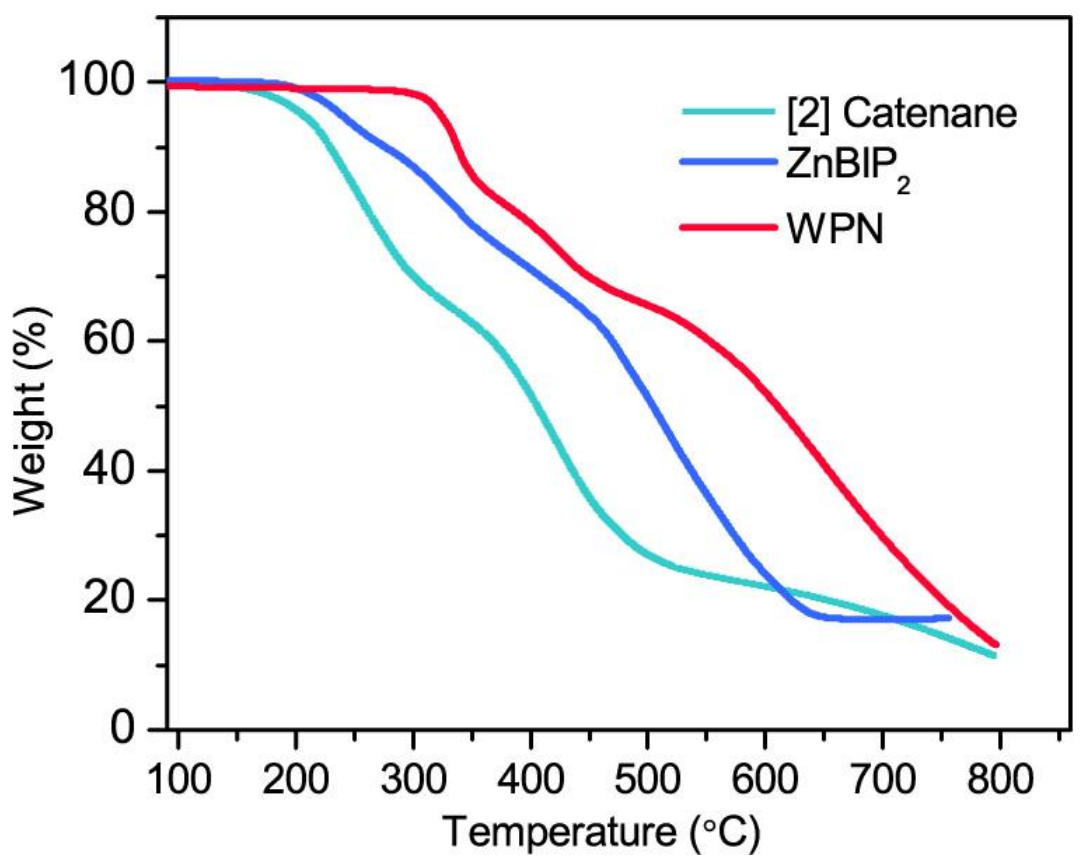

Figure S16. Stack plots of TGA curves of the [2]catenane, $\mathrm{ZnBIP}_{2}$ and WPN. 


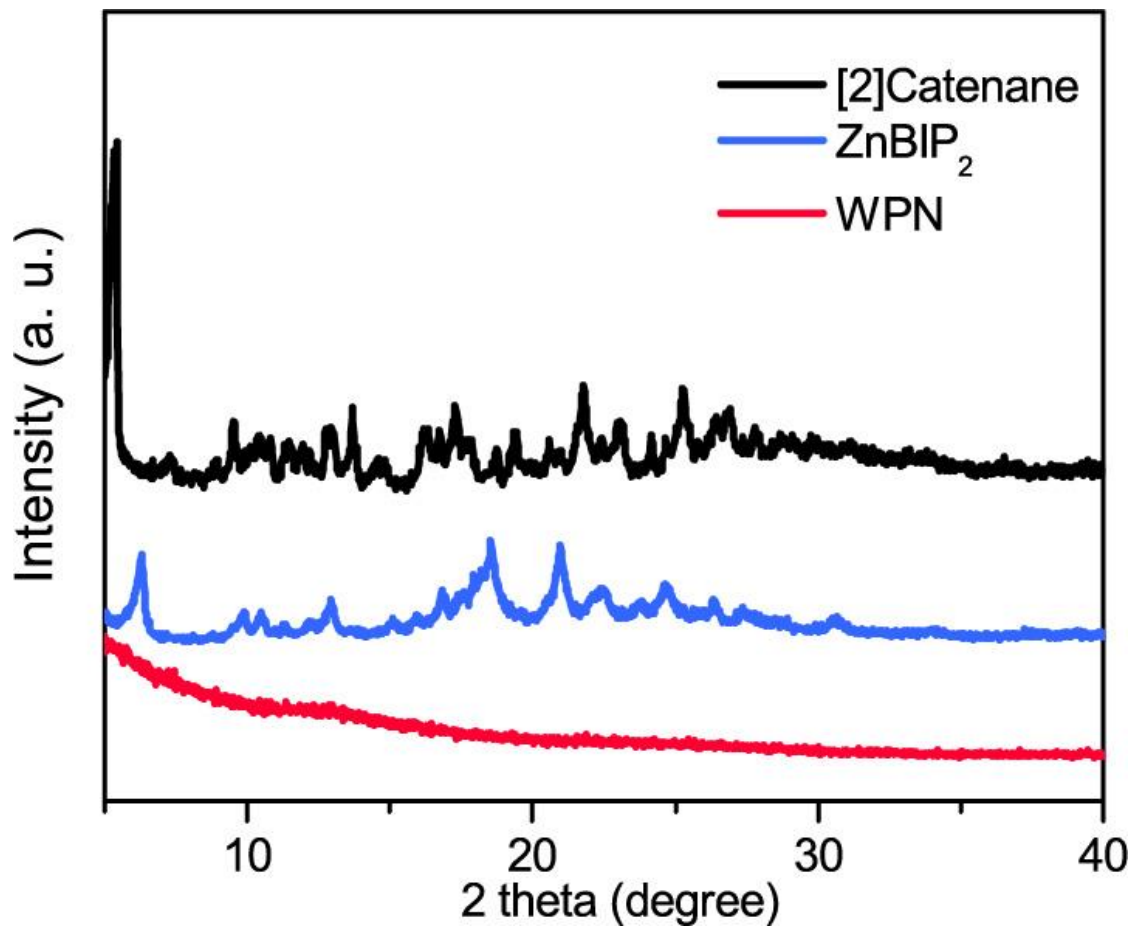

Figure S17. Stack plots of XRD patterns of the [2] catenane, $\mathrm{ZnBIP}_{2}$ and WPN. 
11. Fluorescence emission spectra of the compound $5\left(\mathrm{ZnBIP}_{2}\right)$ in $\mathrm{CH}_{2} \mathrm{Cl}_{2} /$ hexane mixtures with different hexane contents

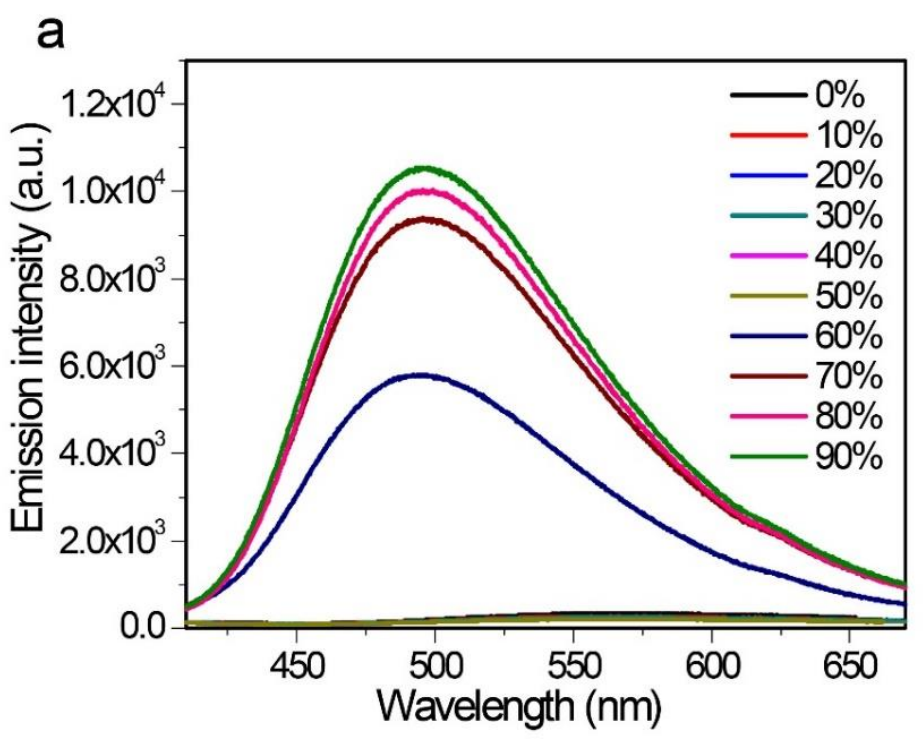

b

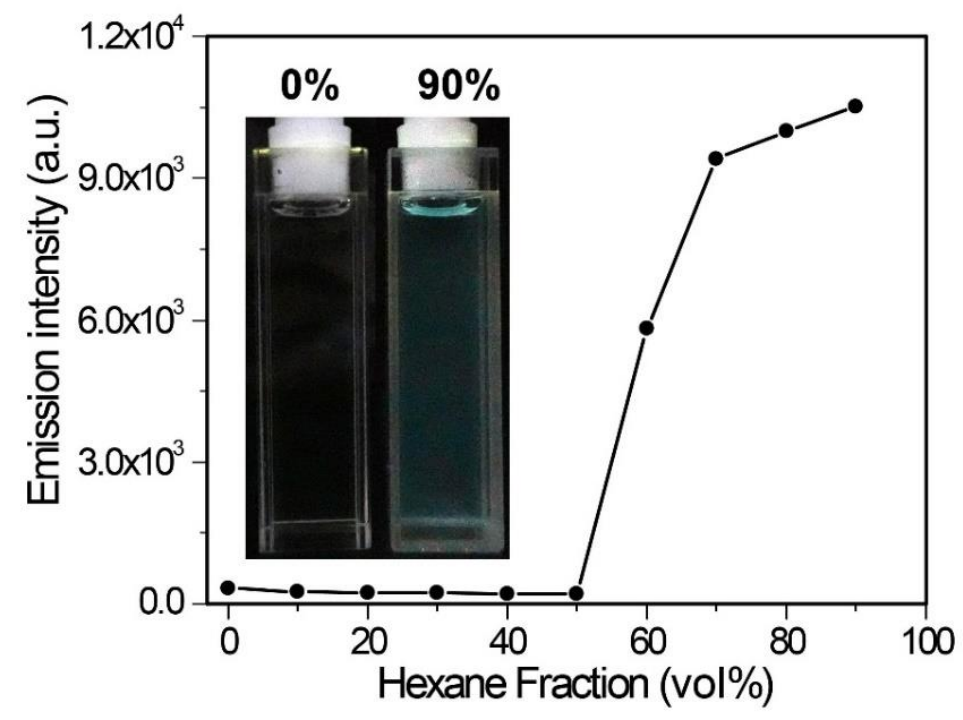

Figure S18. Fluorescence emission spectra (a) and plot of maximum emission intensity (b) of compound $5\left(\mathrm{ZnBIP}_{2}\right)$ versus hexane fraction in $\mathrm{CH}_{2} \mathrm{Cl}_{2} /$ hexane mixtures $\left(\lambda_{\mathrm{ex}}=320 \mathrm{~nm}, c=10.0 \mu \mathrm{M}\right)$. Insets: photographs of 5 in $0 \% / 90 \%$ $\mathrm{CH}_{2} \mathrm{Cl}_{2} /$ hexane mixture upon excitation at $365 \mathrm{~nm}$ using an UV lamp at room temperature $(c=10.0 \mu \mathrm{M})$. 
12. Photographs of compound 5 (ZnBIP2) in $\mathrm{CH}_{2} \mathrm{Cl}_{2} /$ hexane mixtures with different hexane contents

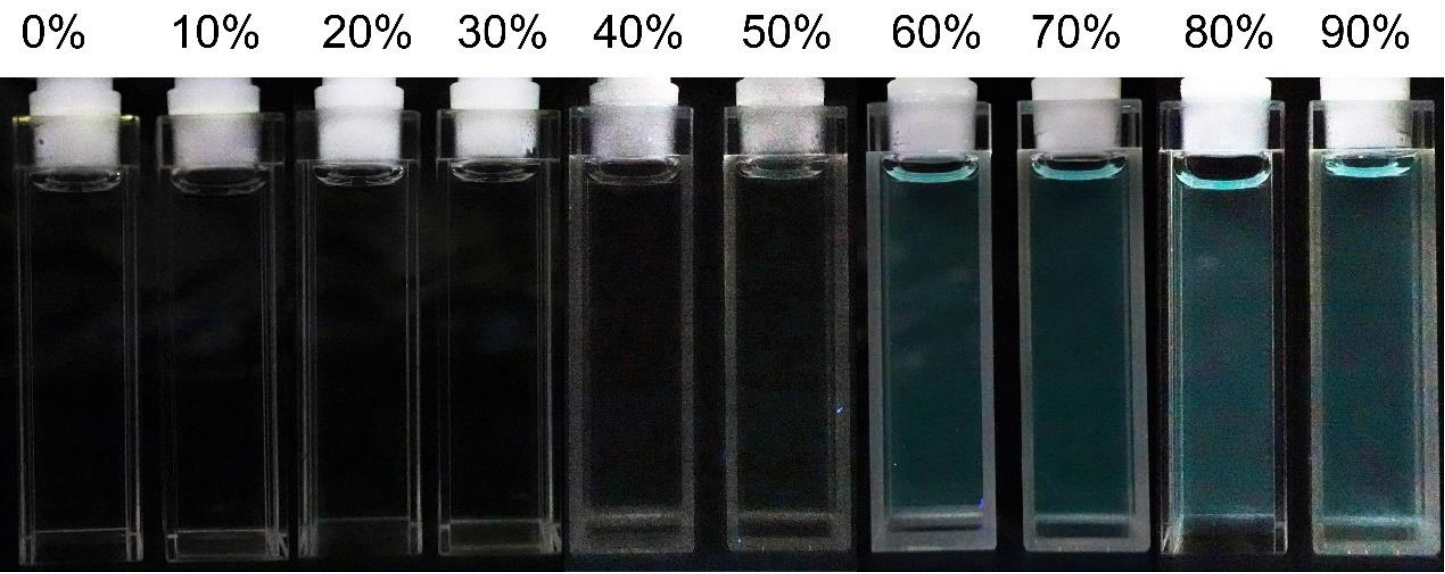

Figure S19. Photographs of compound $5\left(\mathrm{ZnBIP}_{2}\right)$ in $\mathrm{CH}_{2} \mathrm{Cl}_{2} /$ hexane mixtures with different hexane contents $(c=10.0 \mu \mathrm{M})$.

13. Fluorescence emission spectra of the [2]catenane in $\mathrm{CH}_{2} \mathrm{Cl}_{2}$ /hexane mixtures with different hexane contents

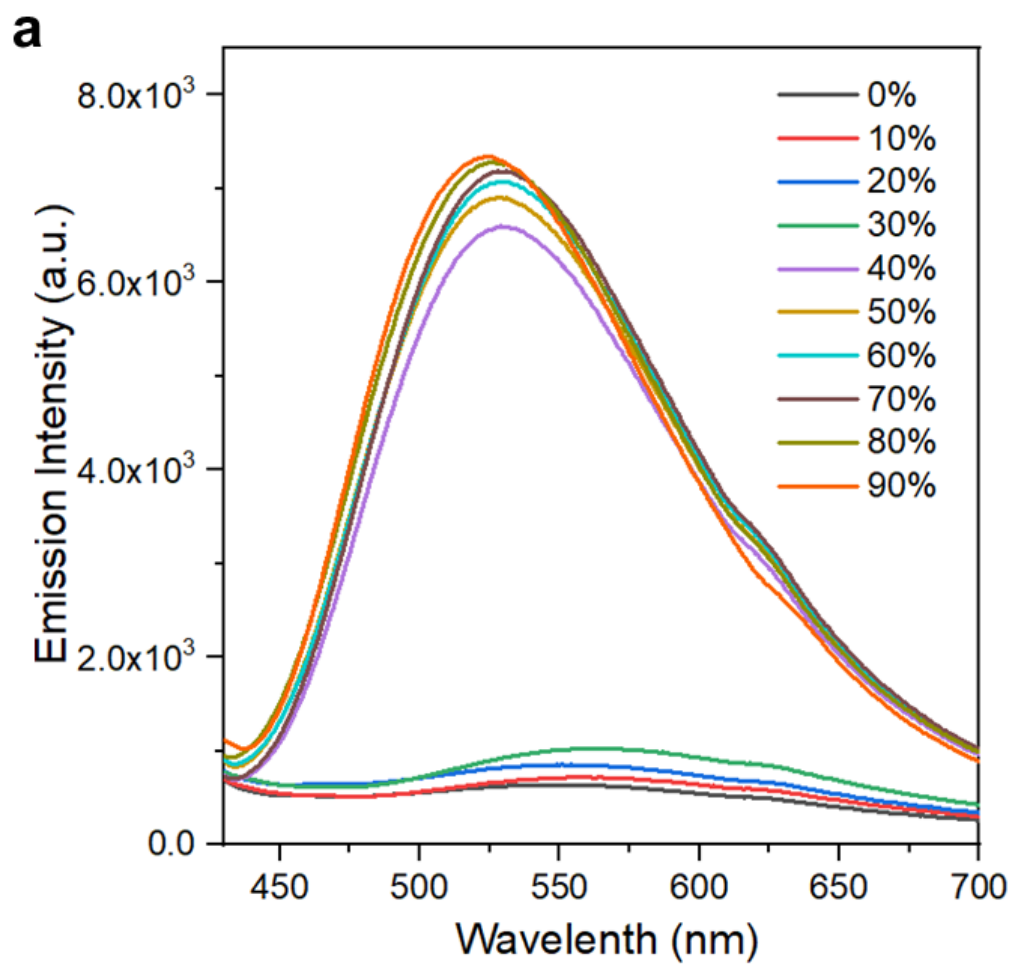




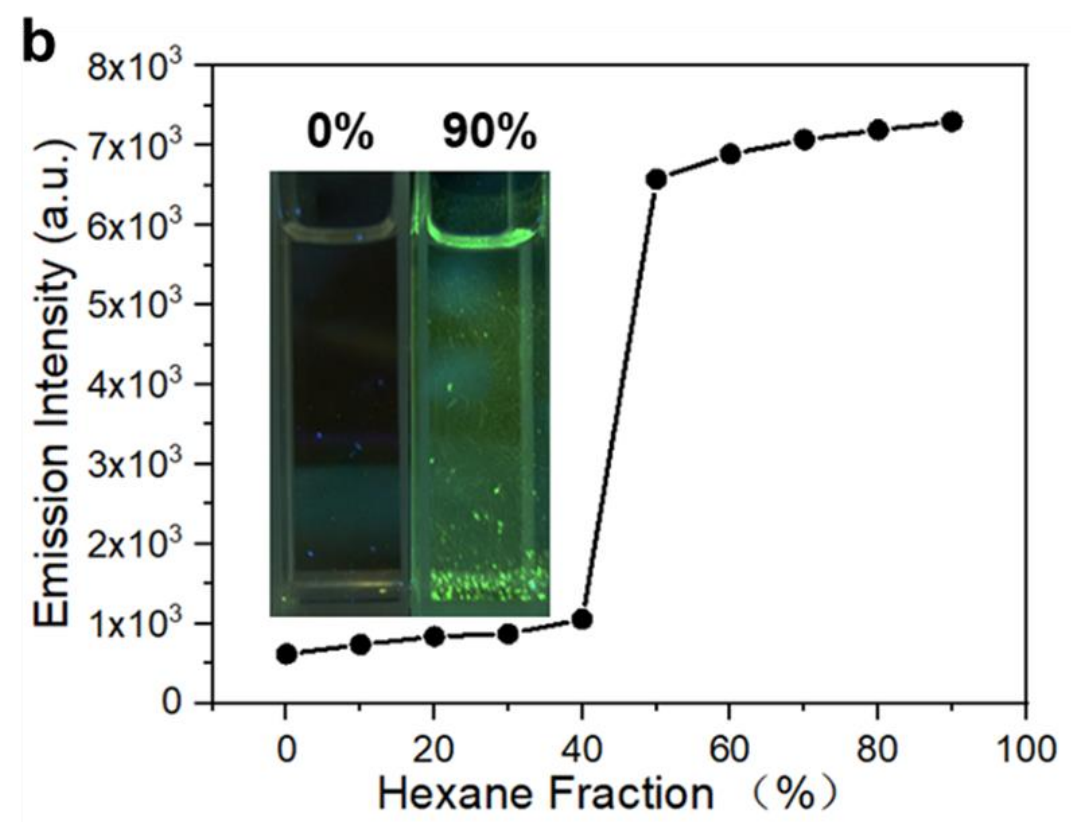

Figure S20. Fluorescence emission spectra (a) and plot of maximum emission intensity (b) of the [2] catenane versus hexane fraction in $\mathrm{CH}_{2} \mathrm{Cl}_{2} /$ hexane mixtures $\left(\lambda_{\mathrm{ex}}\right.$ $=360 \mathrm{~nm}, c=10.0 \mu \mathrm{M})$. Insets: photographs of the [2]catenane in $0 \% / 90 \%$ $\mathrm{CH}_{2} \mathrm{Cl}_{2} /$ hexane mixture upon excitation at $365 \mathrm{~nm}$ using an $\mathrm{UV}$ lamp at room temperature $(c=10.0 \mu \mathrm{M})$. 
14. $U V$-vis spectra, quantum yields and fluorescence lifetimes of the compound $5\left(\mathrm{ZnBIP} \mathrm{P}_{2}\right)$, [2]catenane and WPN

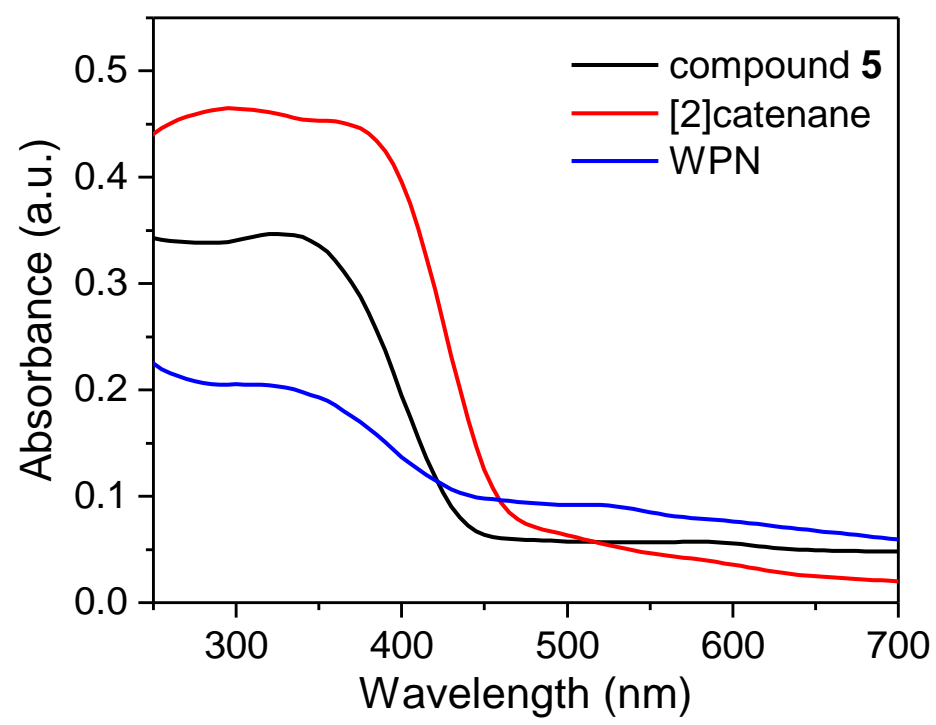

Figure S21. Absorption spectra of the compound $5\left(\mathrm{ZnBIP}_{2}\right)$, [2]catenane and WPN in the solid state.

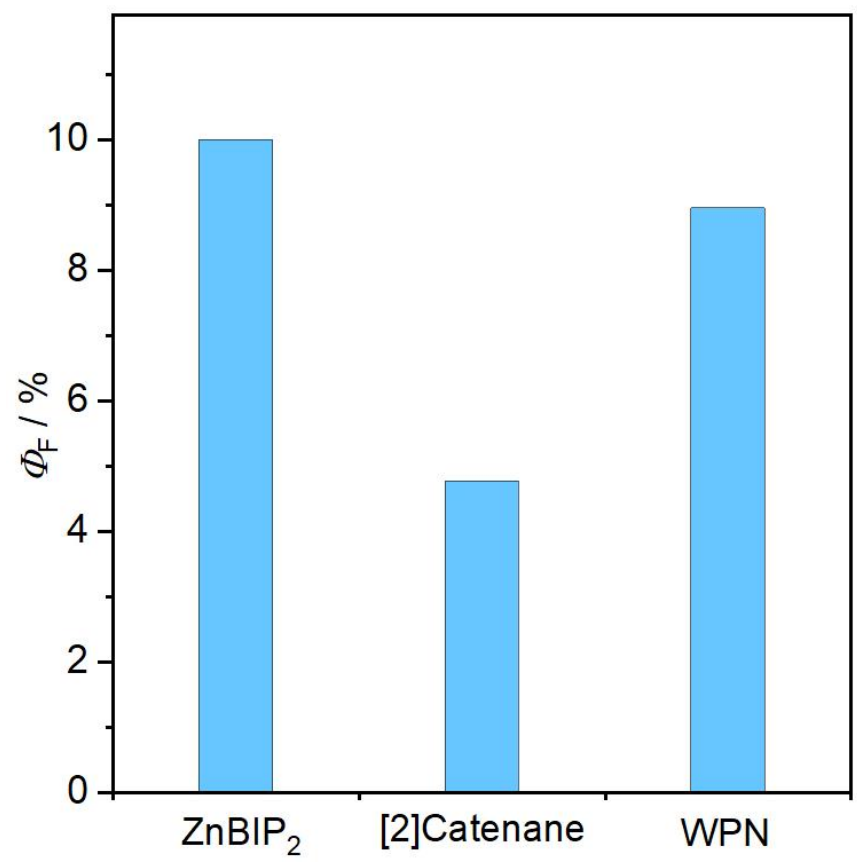

Figure S22. Quantum yields of the compound $5\left(\mathrm{ZnBIP}_{2}\right)$, [2]catenane and WPN in the solid state. 


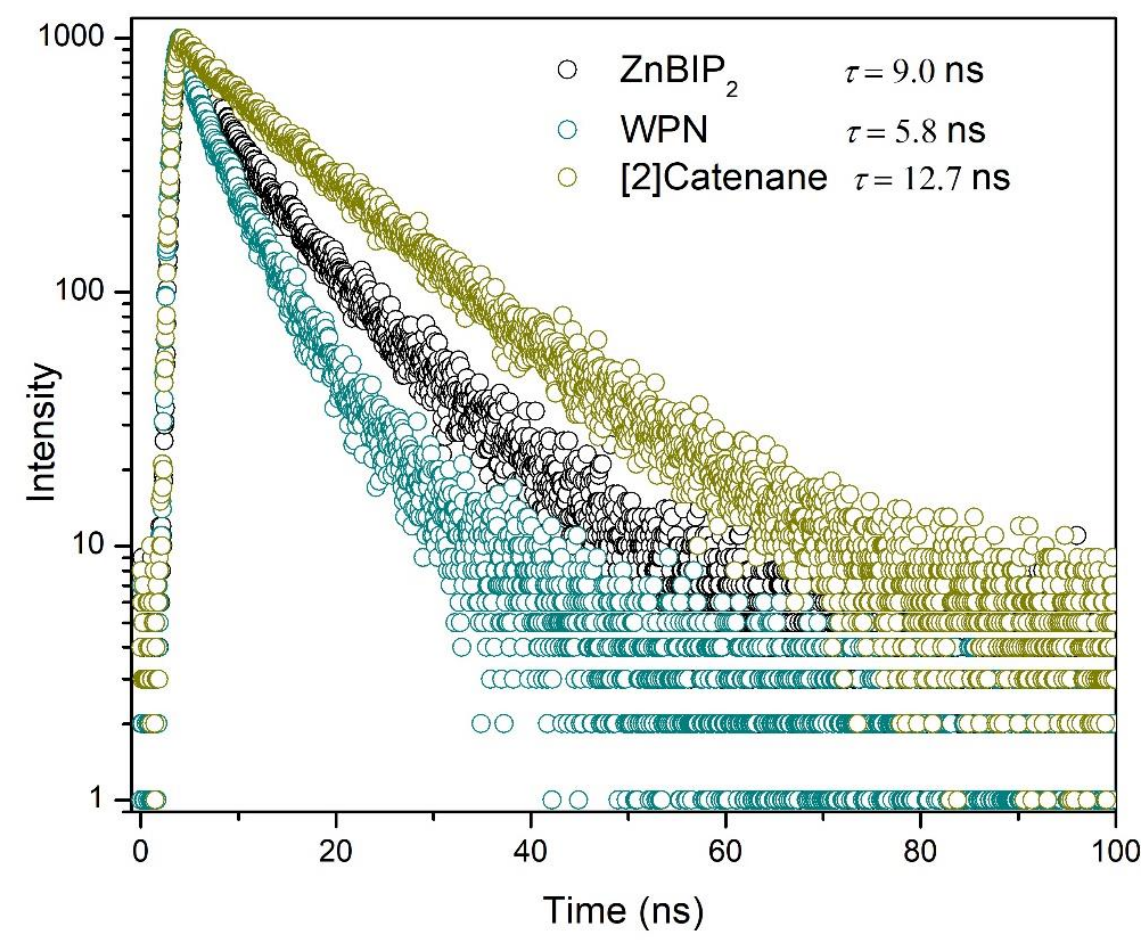

Figure S23. Fluorescence decay profiles of the compound $5\left(\mathrm{ZnBIP}_{2}\right)$, [2]catenane and WPN in the solid state. 
a

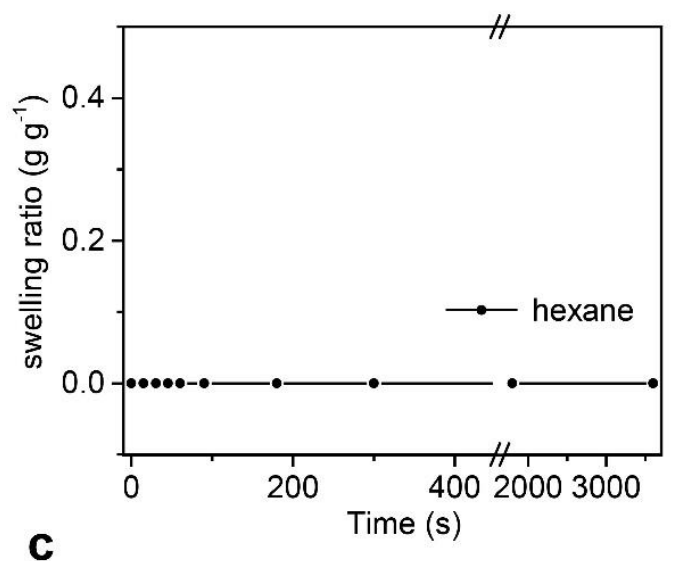

C
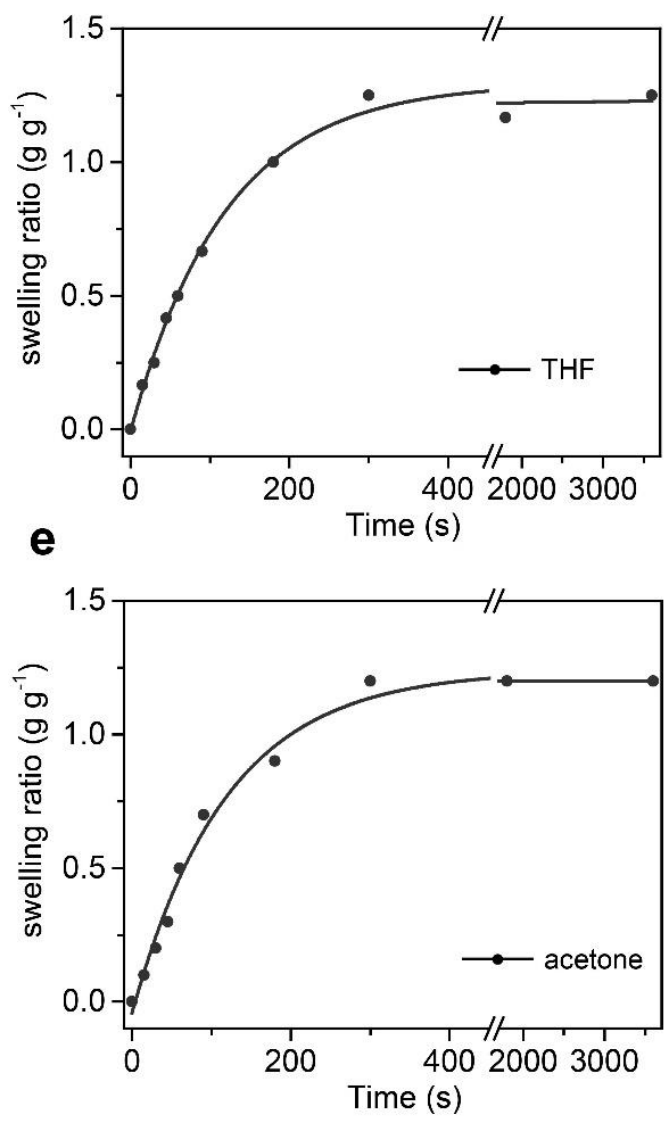

b
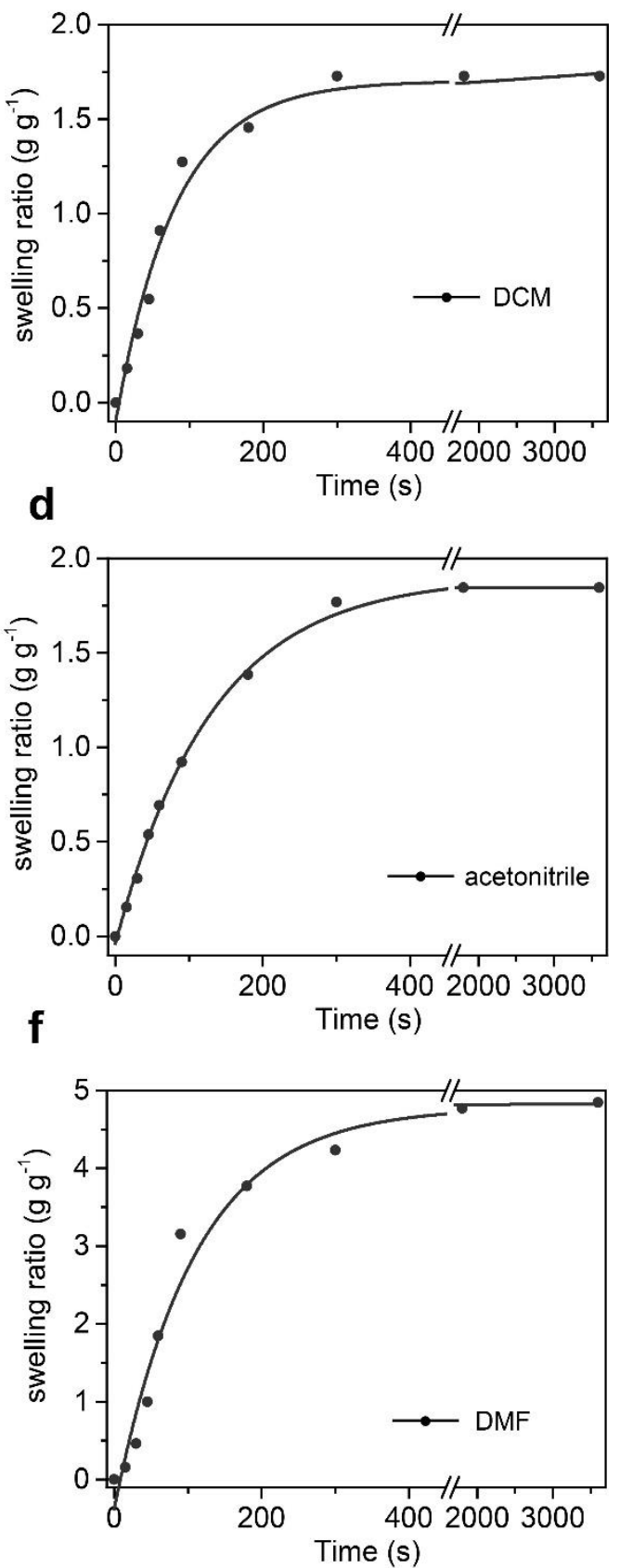

Figure S24. Swelling ratios of the WPN in (a) hexane, (b) dichloromethane (DCM), (c) tetrahydrofuran (THF), (d) acetonitrile, (e) acetone and (f) dimethyl formamide (DMF) as a function of the swelling time. The experiments were performed at room temperature by soaking $~ 5.0 \mathrm{mg}$ sample in $10 \mathrm{~mL}$ solvent. 


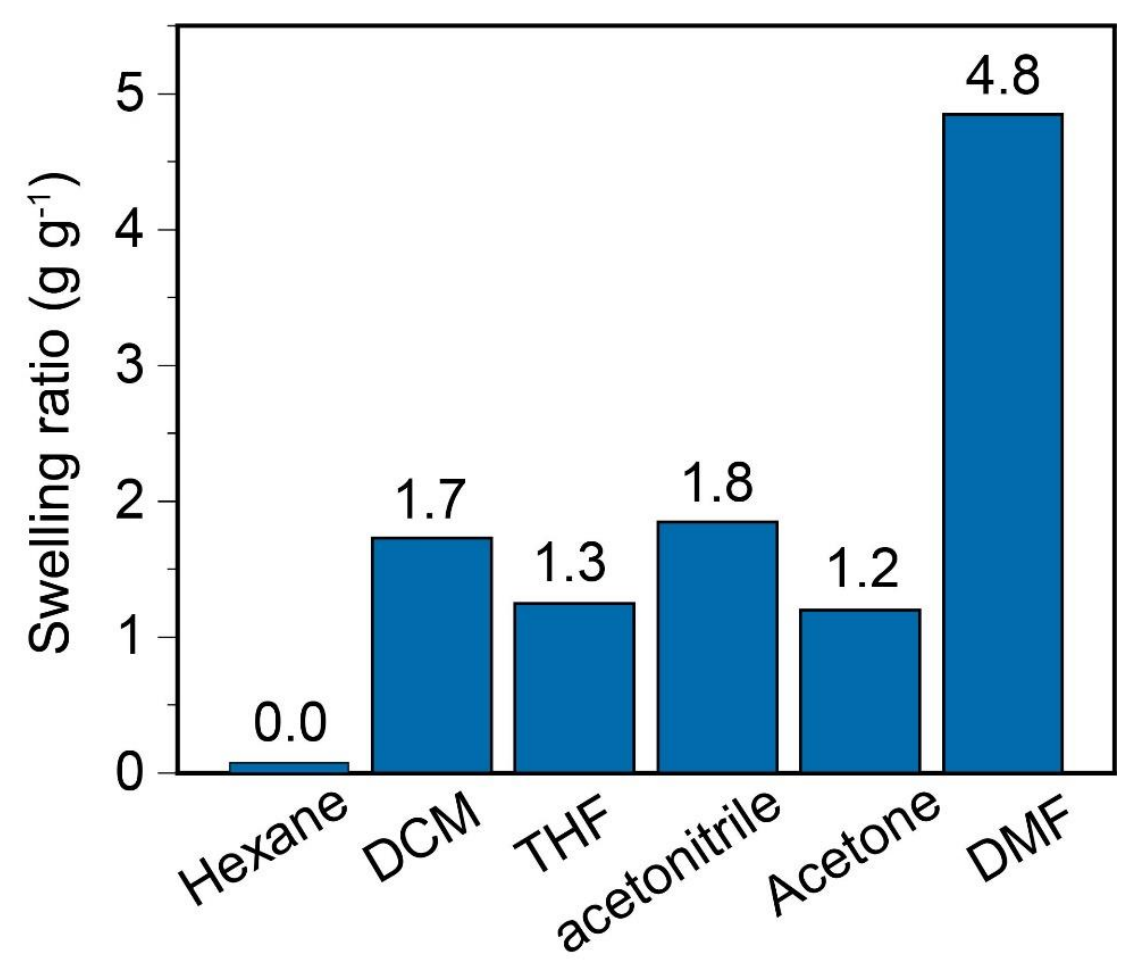

Figure S25. Swelling ratios of the WPN in different solvents.

To determine the swelling property of the WPN, square samples were immersed in different solvents at room temperature. After different times, the samples were taken out and the residual solvent was carefully wiped out to test the mass of the swollen samples. The results at $1 \mathrm{~h}$ were used to calculate the equilibrium swelling degree (Q) using the following equation: ${ }^{\mathrm{S} 2}$

$$
Q=\frac{w_{t}-w_{0}}{w_{0}}
$$

where $\mathrm{w}_{\mathrm{t}}$ is the weight of the swollen sample at time $t$, and $\mathrm{w}_{0}$ is the weight of the dry sample. 
16. Mechanical properties of the demetalated WPN.
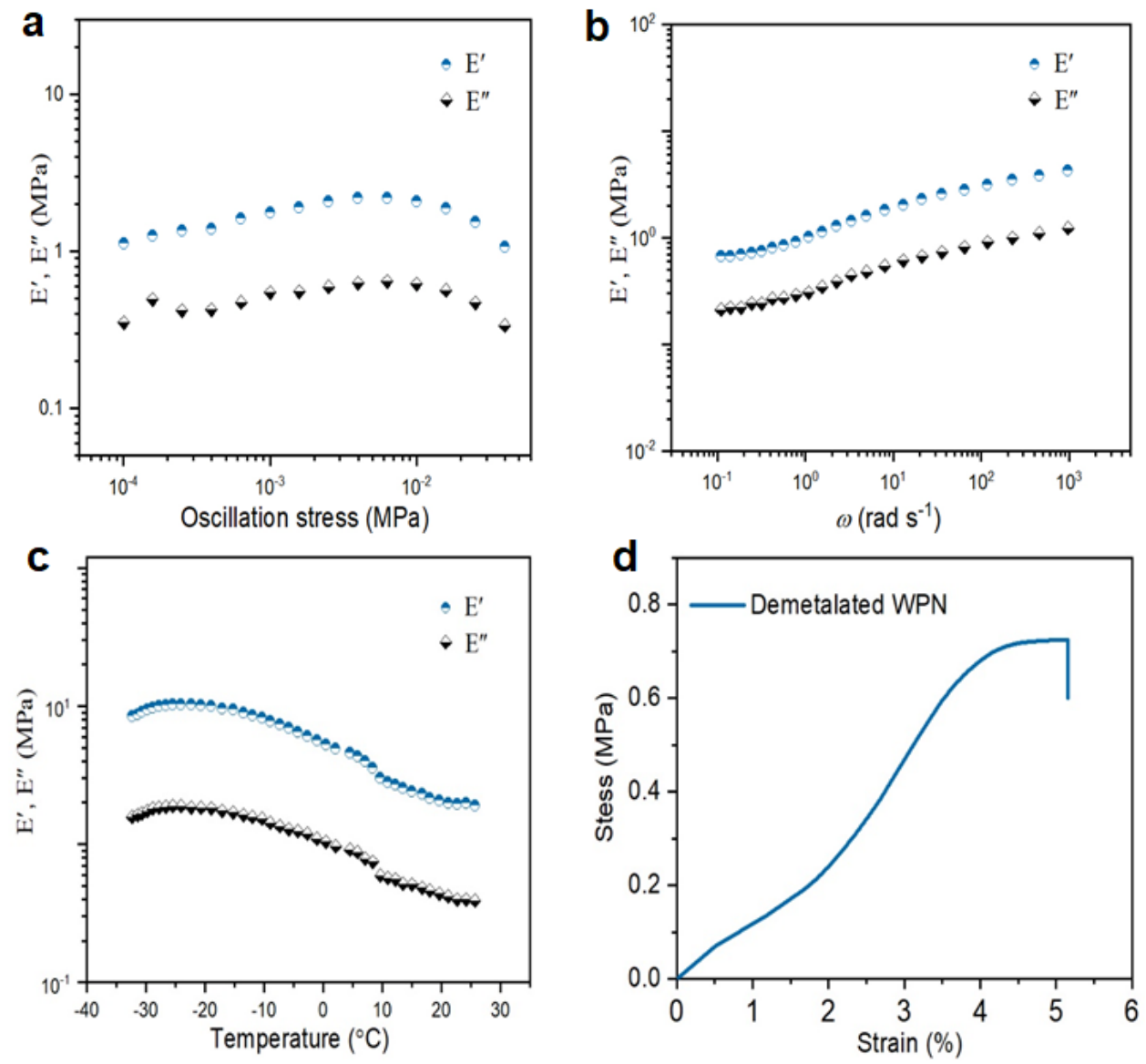

Figure S26. (a) Storage modulus $\left(E^{\prime}\right)$ and loss modulus $\left(E^{\prime \prime}\right)$ versus oscillation stress of the demetalated WPN. (b) $E^{\prime}$ and $E^{\prime \prime}$ versus frequency of the demetalated WPN. (c) $E^{\prime}$ and $E^{\prime \prime}$ versus temperature of the demetalated WPN (d) Stress-strain curve of the demetalated WPN. 
17. Mechanical properties of the remetalated WPN.
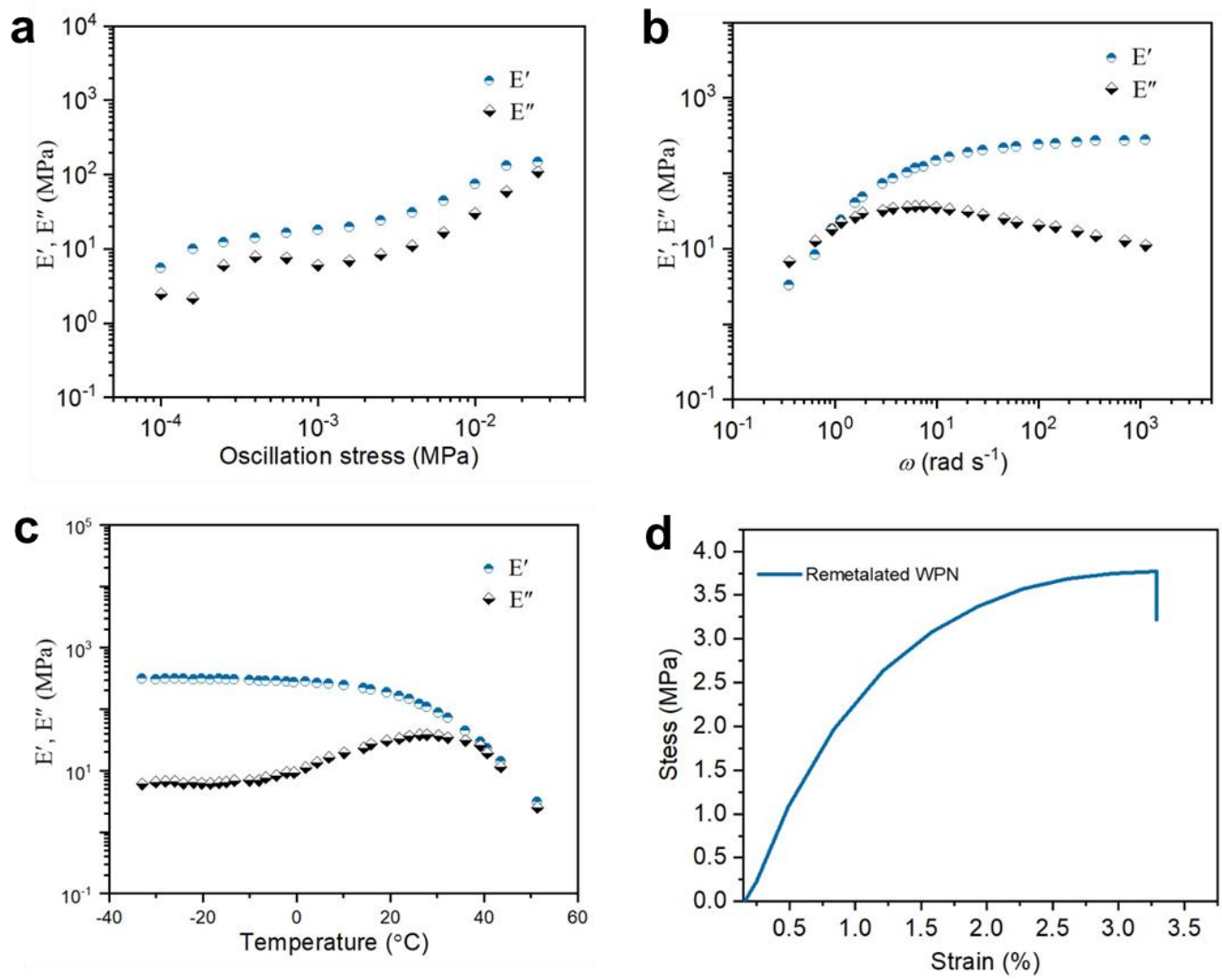

Figure S27. (a) Storage modulus $\left(E^{\prime}\right)$ and loss modulus $\left(E^{\prime \prime}\right)$ versus oscillation stress of the remetalated WPN. (b) $E^{\prime}$ and $E^{\prime \prime}$ versus frequency of the remetalated WPN. (c) $E^{\prime}$ and $E^{\prime \prime}$ versus temperature of the remetalated WPN. (d) Stress-strain curve of the remetalated WPN. 

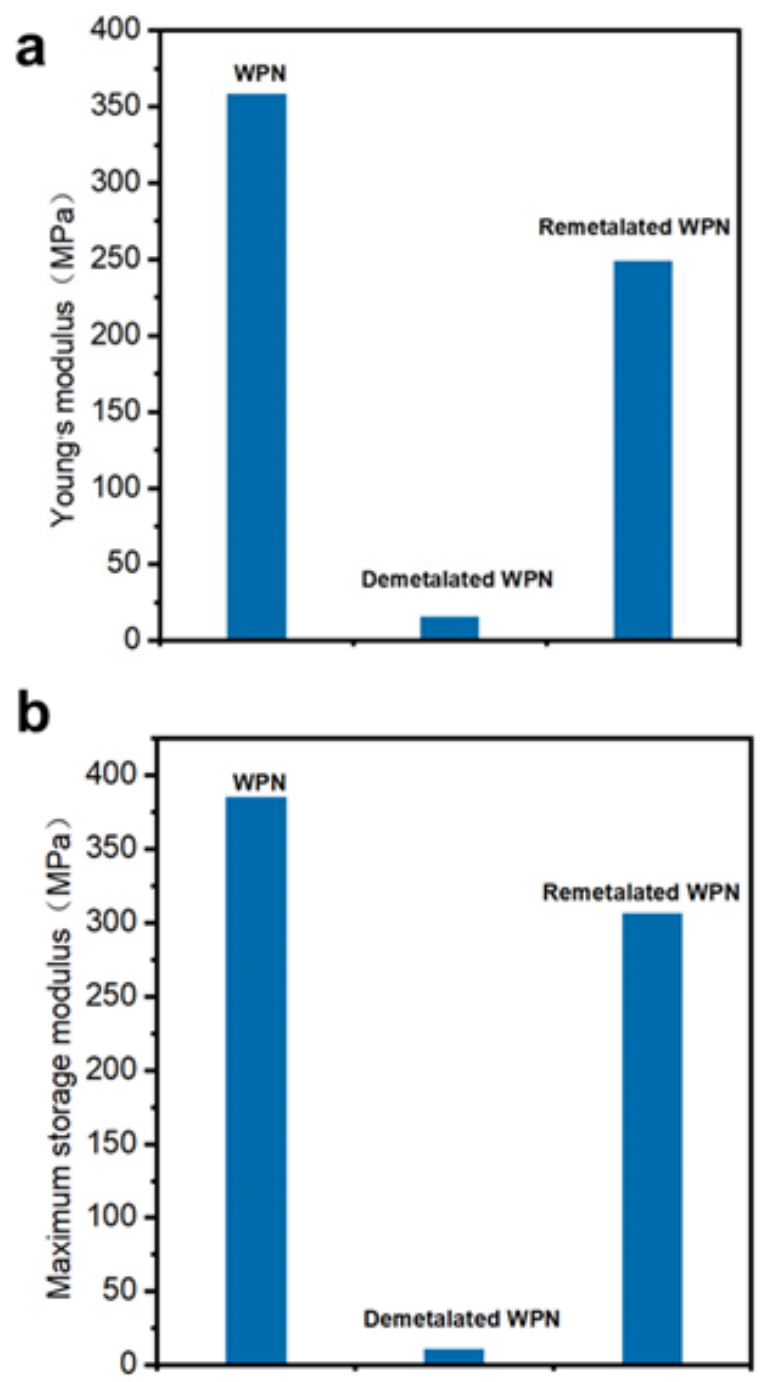

Figure S28. Young's modulus (a) and maximum storage modulus (b) of the WPN, demetalated WPN and remetalated WPN. 
18. AFM nanomechanical mapping tests
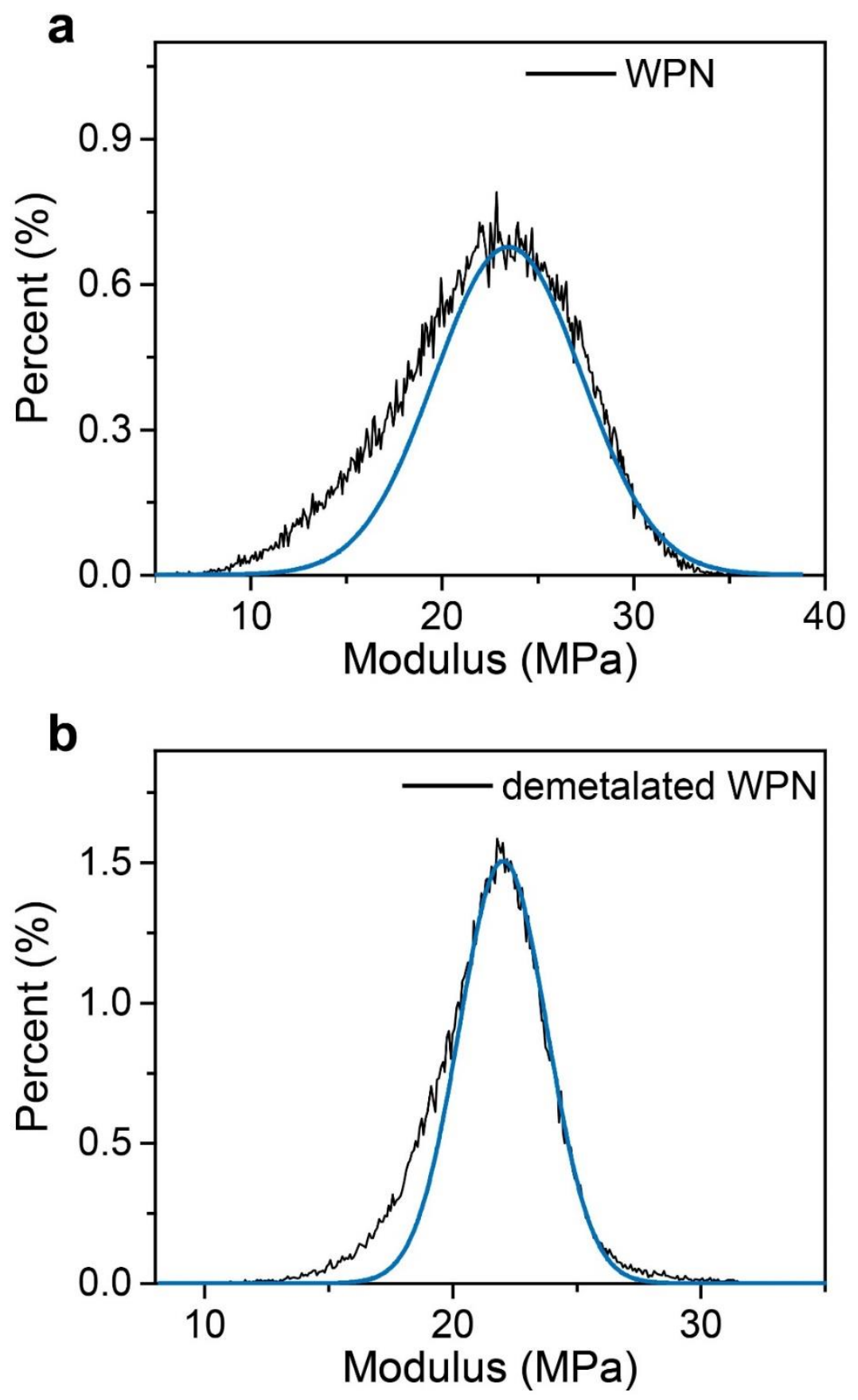


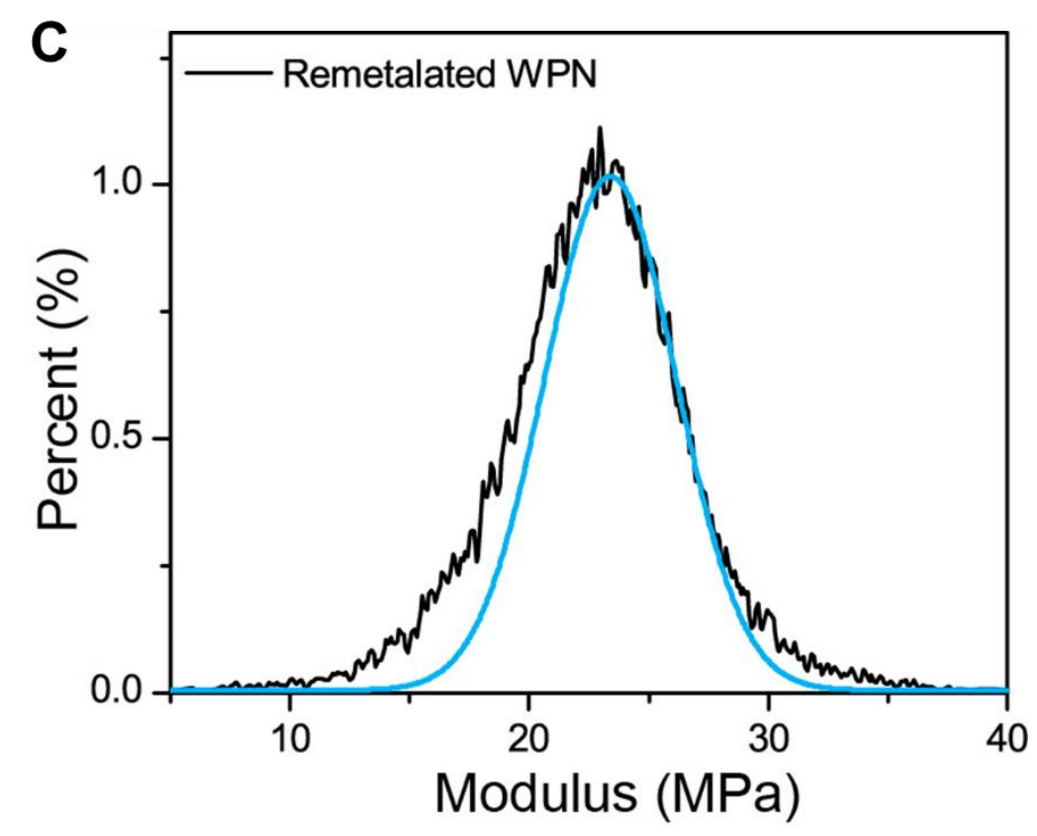

Figure S29. AFM modulus distribution profiles (black lines) and the Gaussian distribution fitting results (blue lines) of the WPN (a), demetalated WPN (b) and remetalated WPN(c).

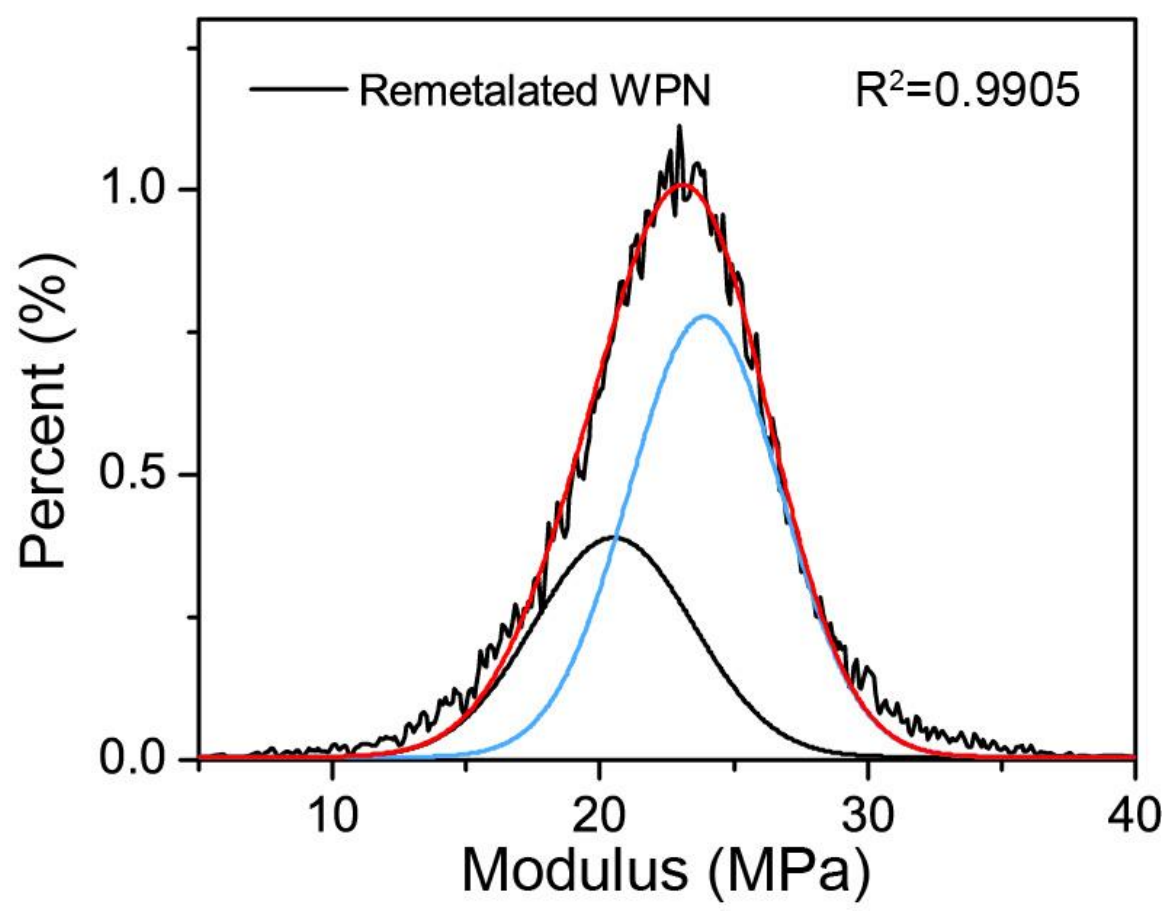

Figure S30. AFM modulus distribution profiles and Gaussian fitting results of the remetalated WPN. 

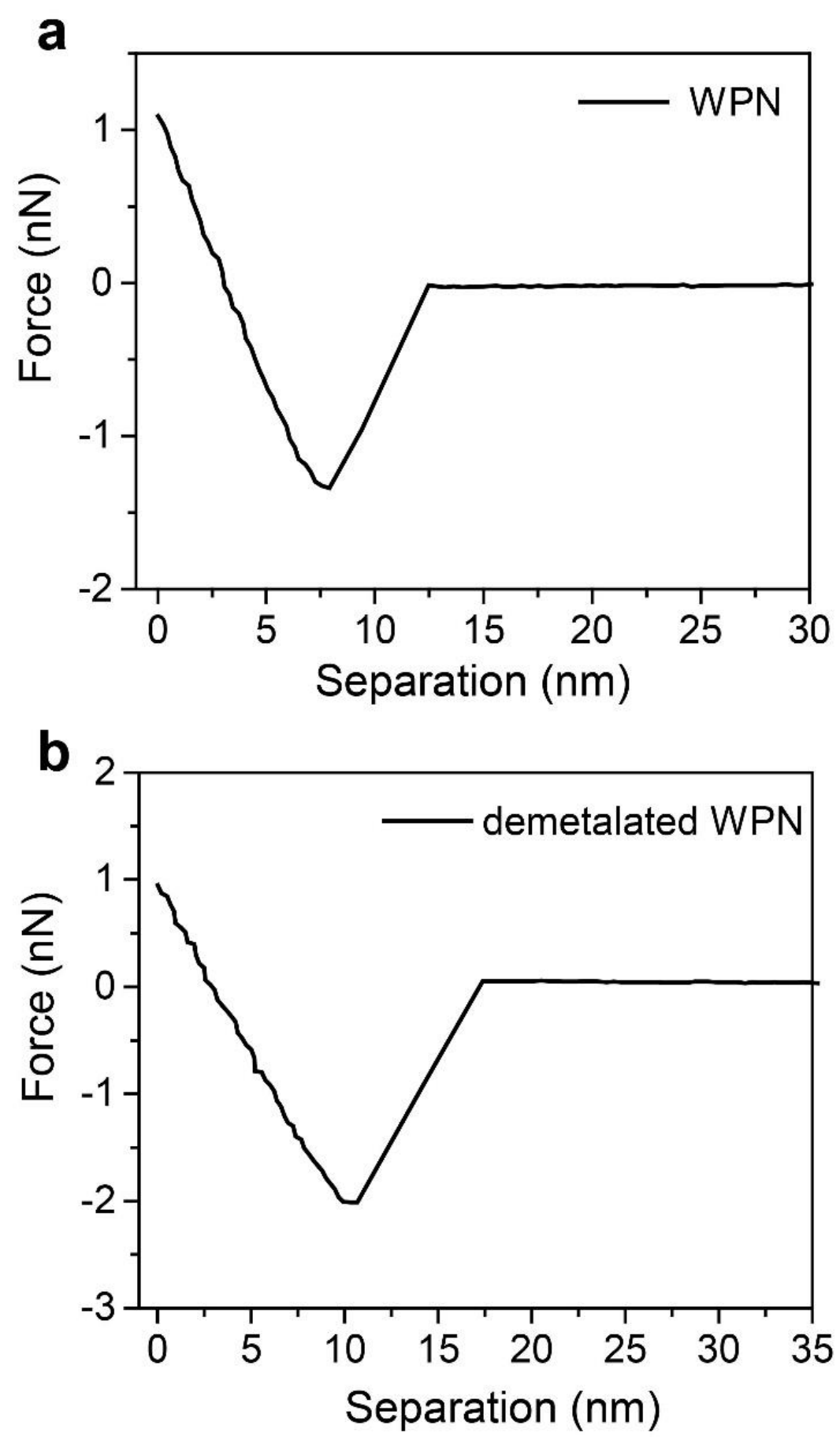

Figure S31. Force-indentation curve extended from the PeakForce maps on WPN (a) and demetalated WPN (b).

Figure S31 shows typical PeakForce micrographs, where both modulus images and the corresponding Young's modulus maps were obtained. The elastic modulus of both WPN and demetalated WPN were determined by fitting the extending portion of each force-indentation 
curve with the Derjaguin-Muller-Toporov (DMT) model, ${ }^{\mathrm{S} 3}$ which can be applied in the case of materials displaying low adhesion and using a tip with a relatively small radius. The reduced modulus $\left(E^{*}\right)$ obtained is expressed by the formula:

$$
F=\frac{4}{3} E^{*} \sqrt{R d^{3}}+F_{a d s}
$$

where $F$ is the applied force, $R$ is the tip radius, $\mathrm{d}$ corresponds to the sample deformation and $F_{\text {ads }}$ refers to the maximum adhesion force. The relation between $E^{*}$ and the Young's modulus $(E)$ is:

$$
\frac{1}{E^{*}}=\frac{1-v_{t}^{2}}{E_{t}}+\frac{1-v^{2}}{E}
$$

where $v_{\mathrm{t}}$ and $E_{\mathrm{t}}$ are the Poisson's ratio and the elastic modulus of the tip, and $v$ and $E$ are the Poisson's ratio and the Young's modulus of the sample. The Young's modulus of the WPN (Figure S31a) and demetalated WPN (Figure S31b) are 24.3 and $20.4 \mathrm{MPa}$, respectively.

\section{Coarse-grained simulation}

The Coarse-grained (CG) molecular model is developed for $\mathrm{ZnBIP}_{2}$ to capture the necessary features of molecular structure of $\mathrm{ZnBIP}_{2}$. The CG model of $\mathrm{ZnBIP}_{2}$ is shown in Figure $\mathrm{S} 32$ in which the size of beads is $2 \sigma$. In order to simulate the bonding of $\mathrm{ZnBIP}_{2}$ during ROMP, the patch beads with diameter $0.2 \sigma$ are placed at the end of alkene chains. The masses of beads and patches are unit. The bond length $(b)$ stretch and bond angle $(\theta)$ interactions are described using harmonic functions

$$
\begin{aligned}
& U^{\text {bond }}(b)=k_{\mathrm{b}}\left(b-b_{0}\right)^{2} \\
& U^{\text {angle }}(\theta)=k_{\mathrm{a}}\left(\theta-\theta_{0}\right)^{2}
\end{aligned}
$$

where $k_{\mathrm{b}}=100 \sigma^{-2}$ and $k_{\mathrm{a}}=100 \operatorname{rad}^{-2}$ to keep the relative rigidity of the CG model of $\mathrm{ZnBIP}_{2}$. The intermolecular interaction between beads is modelled by Weeks-Chandler-Andersen potential,

$$
U^{\mathrm{WCA}}\left(r_{i j}\right)= \begin{cases}U^{L J}\left(r_{i j}\right)-U^{L J}\left(r_{c}\right) & r_{i j}<2.24 \sigma \\ 0 & r_{i j} \geq 2.24 \sigma\end{cases}
$$

$U^{\mathrm{LJ}}\left(r_{i j}\right)$ is Lennard-Jones potential, and $r_{i j}$ is the inter-bead separation. The bonding is modelled as strong attractions between patch beads via $U^{\mathrm{LJ}}\left(r_{i j}\right)$ is shifted Lennard-Jones 
potential with interaction strength $\epsilon_{\mathrm{LJ}}=20 \epsilon$ and cutoff $r_{c}=2.0 \sigma$. No interaction is allowed between bead and patch.
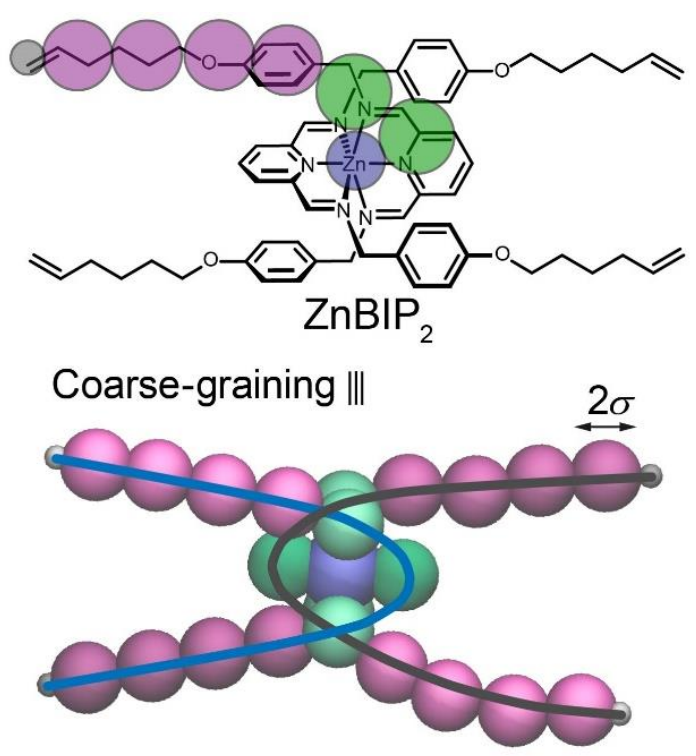

Figure S32. Coarse-grained model of $\mathrm{ZnBIP}_{2}$

Coarse-grained molecular simulation is performed in the LAMMPS ${ }^{\mathrm{S} 4}$ code using Langevin dynamics which is widely used to model condensed behaviour of soft material. ${ }^{\mathrm{s} 5, \mathrm{~s} 6}$ The simulation trajectory is computed according to

$$
m \frac{\mathrm{d}^{2} \mathbf{r}}{\mathrm{d} t^{2}}=-\nabla U(\mathbf{r})-\gamma \cdot \mathbf{v}+\mathbf{F}_{R}
$$

where the random force $\mathbf{F}_{R}$ mimics the Brownian effect of solvent molecules on the solutes. The dimensionless unit are used throughout the simulation in which time unit $\tau=\sqrt{m \sigma^{2} / \epsilon}$. The initial configuration is built using $\mathrm{Packmol}^{\mathrm{S} 7}$ containing $600 \mathrm{ZnBIP}_{2}$ monomers with dimensions $14.5 \times 14.5 \times 14.5 \mathrm{~nm}^{3}$. The simulations are run for at least $10^{8}$ time steps for equilibration and further $2 \times 10^{8}$ time steps are needed to produce trajectory for analysis. The simulations are performed for sufficiently long to ensure the system reach equilibrium state configuration. The result of simulation is visualized using VMD software. ${ }^{\text {s8 }}$

Notably, we compute the bonding of the $\mathrm{ZnBIP}_{2}$ averaged over simulation trajectory, and find the tri-substituted alkenes account for about $5 \%, 47 \%$ is pair-bonding and $48 \%$ for non-bonded patch beads. The presence of tri-substituted alkenes is caused by possible multiple attractions of patch beads, which can be prevented due to steric repulsion in the current study, but cannot be fully avoided in theory. ${ }^{\mathrm{S} 5}$ Such molecular model is widely used in 
simulation studies of complex phenomena of soft matter such as DNA hydrogel ${ }^{\mathrm{S} 5}$ and POSS-polymer composites. ${ }^{\mathrm{S} 6}$ 


\section{References:}

S1. Leigh, D. A.; Lusby, P. J.; Teat, S. J.; Wilson, A. J.; Wong, J. K. Y. Angew. Chem. Int. Ed. 2001, $40,1538-1543$.

S2. Hoshino, K.; Nakajima, T.; Matsuda, T.; Sakai, T.; Gong, J. P. Soft Matter 2018, 14, 9693-9701.

S3. Cappella, B.; Dietler, G. Surf. Sci. Rep. 1999, 34, 1-104.

S4. Plimpton, S. J. Comput. Phys. 1995, 117, 1-19.

S5. Xing, Z.; Ness, C.; Frenkel, D.; Eiser, E. Macromolecules 2019, 52, 504-512.

S6. Yue, K.; Huang, M.; Marson, R. L.; He, J.; Huang, J.; Zhou, Z.; Wang, J.; Liu, C.; Yan, X.; Wu, K.;

Guo, Z.; Liu, H.; Zhang, W.; Ni, P.; Wesdemiotis, C.; Zhang, W.-B.; Glotzer, S. C.; Cheng, S. Z. D. Proc. Nat. Acad. Sci. U.S.A. 2016, 113, 14195-14200.

S7. Martínez, L.; Andrade, R.; Birgin, E. G.; Martínez, J. M. J. Comput. Chem. 2009, 30, 2157-2164.

S8. Humphrey, W.; Dalke, A.; Schulten, K. J. Mol. Graph. 1996, 14, 33-38. 